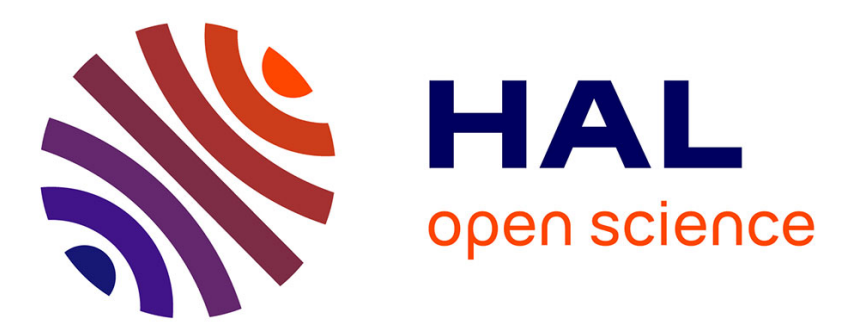

\title{
Complex patterns of spatially extended generators of epileptic activity: Comparison of source localization methods cMEM and 4-ExSo-MUSIC on high resolution EEG and MEG data
}

A. Chowdhury, Isabelle Merlet, G. Birot, E. Kobayashi, A. Nica, A. Biraben, F. Wendling, J.M. Lina, Laurent Albera, C. Grova

\section{To cite this version:}

A. Chowdhury, Isabelle Merlet, G. Birot, E. Kobayashi, A. Nica, et al.. Complex patterns of spatially extended generators of epileptic activity: Comparison of source localization methods cMEM and 4-ExSo-MUSIC on high resolution EEG and MEG data. NeuroImage, 2016, 143, pp.175 - 195. 10.1016/j.neuroimage.2016.08.044 . hal-01381265

HAL Id: hal-01381265

https://hal-univ-rennes1.archives-ouvertes.fr/hal-01381265

Submitted on 9 Dec 2016

HAL is a multi-disciplinary open access archive for the deposit and dissemination of scientific research documents, whether they are published or not. The documents may come from teaching and research institutions in France or abroad, or from public or private research centers.
L'archive ouverte pluridisciplinaire HAL, est destinée au dépôt et à la diffusion de documents scientifiques de niveau recherche, publiés ou non, émanant des établissements d'enseignement et de recherche français ou étrangers, des laboratoires publics ou privés. 


\title{
Complex patterns of spatially extended generators of epileptic activity: Comparison of source localization methods cMEM and 4-ExSo-MUSIC on High Resolution EEG and MEG data
}

Chowdhury R. A. ${ }^{{ }^{*} 1}$, Merlet I. ${ }^{\mathrm{b}, \mathrm{c} 1}$, Birot G. ${ }^{\mathrm{d}}$, Kobayashi E. ${ }^{\mathrm{e}}$, Nica A. ${ }^{\mathrm{b}, \mathrm{c}, \mathrm{f}}$, Biraben A. ${ }^{\mathrm{b}, \mathrm{c}, \mathrm{f}}$, Wendling F. ${ }^{\mathrm{b}, \mathrm{c}}$, Lina J.M. ${ }^{g}$, Albera L. ${ }^{\mathrm{b}, \mathrm{c}, \mathrm{h}}$, Grova C. ${ }^{\mathrm{a}, \mathrm{e}, \mathrm{i}}$

${ }^{a}$ Multimodal functional imaging lab, Biomedical Engineering Dpt, McGill University , Montreal, Canada

${ }^{\mathrm{b}}$ INSERM, U1099,Rennes, 35000, France

'Université de Rennes 1, Laboratoire de Traitement du Signal et de l'Image, Rennes, 35000, France

${ }^{\mathrm{d} D e p a r t m e n t ~ o f ~ F u n d a m e n t a l ~ a n d ~ C l i n i c a l ~ N e u r o s c i e n c e s, ~ U n i v e r s i t y ~ o f ~ G e n e v a, ~ S w i t z e r l a n d ~}$

${ }^{\mathrm{e}}$ Neurology and neurosurgery department, Montreal Neurological Institute (MNI), McGill University, Montreal, Canada

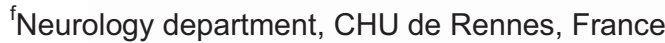

${ }^{9}$ Département de Genie Electrique, Ecole de Technologie Supérieure, Canada,

hINRIA, Centre Inria Rennes - Bretagne Atlantique, 35042 Rennes Cedex, France

'Physics Dpt., PERFORM Centre, Concordia University, Canada

"Corresponding author. Multimodal Functional Imaging Laboratory, Biomedical Engineering Department, McGill University, 3775, rue University, room 332, Montréal, QC, H3A 2B4, Canada. Tel.: 1 (514)3981678; fax: 1(514)398-7461. rasheda.chowdhury@mail.mcgill.ca

Keywords: EEG/MEG source localization, interictal epileptic discharges, neural mass model, maximum entropy on the mean, 4-ExSo-MUSIC, higher order statistics

\begin{abstract}
Electric Source Imaging (ESI) and Magnetic Source Imaging (MSI) of EEG and MEG signals are widely used to determine the origin of interictal epileptic discharges during the pre-surgical evaluation of patients with epilepsy. Epileptic discharges are detectable on EEG/ MEG scalp recordings only when associated with a spatially extended cortical generator of several square centimeters, therefore it is essential to assess the ability of source localization methods to recover such spatial extent.

In this study we evaluated two source localization methods that have been developed for localizing spatially extended sources using EEG/MEG data: coherent Maximum Entropy on the Mean (cMEM) and 4th order Extended Source
\end{abstract}

\footnotetext{
${ }^{1}$ Rasheda Arman Chowdhury and Isabelle Merlet contributed equally to this manuscript.
} 
Multiple Signal Classification (4-ExSo-MUSIC). In order to propose a fair comparison of the performances of the two methods in MEG versus EEG, this study considered realistic simulations of simultaneous EEG/MEG acquisitions taking into account an equivalent number of channels in EEG (257 electrodes) and MEG (275 sensors), involving a biophysical computational neural mass model of neuronal discharges and realistically shaped head models. cMEM and 4-ExSo-MUSIC were evaluated for their sensitivity to localize complex patterns of epileptic discharges which includes (a) different locations and spatial extents of multiple synchronous sources, and (b) propagation patterns exhibited by epileptic discharges. Performance of the source localization methods was assessed using a detection accuracy index (Area Under receiver operating characteristic Curve, AUC) and a Spatial Dispersion (SD) metric. Finally, we also presented two examples illustrating the performance of cMEM and 4-ExSo-MUSIC on clinical data recorded using high resolution EEG and MEG.

When simulating single sources at different locations, both 4-ExSo-MUSIC and cMEM exhibited excellent performance (median AUC significantly larger than 0.8 for EEG and MEG), whereas, only for EEG, 4-ExSo-MUSIC showed significantly larger AUC values than CMEM. On the other hand, CMEM showed significantly lower SD values than 4-ExSo-MUSIC for both EEG and MEG. When assessing the impact of the source spatial extent, both methods provided consistent and reliable detection accuracy for a wide range of source spatial extents (source sizes ranging from $3-20 \mathrm{~cm}^{2}$ for MEG and $3-30 \mathrm{~cm}^{2}$ for EEG). For both EEG and MEG, 4-ExSo-MUSIC localized single source of large signal-to-noise ratio better than cMEM. In the presence of two synchronous sources, cMEM was able to distinguish well the two sources (their location and spatial extent), while 4-ExSo-MUSIC only retrieved one of them. cMEM was able to detect the spatio-temporal propagation patterns of two synchronous activities while 4-ExSoMUSIC favored the strongest source activity.

Overall, in the context of localizing sources of epileptic discharges from EEG and MEG data, 4-ExSo-MUSIC and cMEM were found accurately sensitive to the location and spatial extent of the sources, with some complementarities. Therefore, they are both eligible for application on clinical data.

\section{Introduction}

ElectroEncephaloGraphy (EEG) and MagnetoEncephaloGraphy (MEG) have been widely used as a noninvasive technique to identify brain activation patterns associated with normal or pathological processes. Due to their high temporal resolution, these techniques are particularly appropriate for following the time course of activation during fast transients such as evoked potentials, epileptic discharges or specific oscillatory patterns (Lopes da Silva, 2013). In the particular context of epilepsy, interictal epileptic discharges (IEDs), recorded between seizures with MEG and EEG in the form of spikes, spike and waves, sharp waves, provide important information on the spatial organization of the epileptogenic network, provided that they can be localized accurately. It is now admitted that IEDs recruit a rather large area of cortex in a quasi-synchronous manner. Thus, to be detected on surface recordings, a minimum area of 4-8 cm² for EEG (Cooper et al., 1965; Ebersole, 1997; Merlet and Gotman, 1999; Tao et al., 2007; von Ellenrieder et al., 2014) and of 3-4 cm² for MEG (Hari, 1990; Mikuni et al., 1997; Oishi et al., 2002) has been suggested as activated during IEDs. Therefore, not only is it important to localize the origin of 
IEDs but also to recover their spatial extent and estimate the temporal course of their activity. This task can become particularly challenging when several distributed regions with highly synchronized activity are simultaneously active or are involved during a propagation process.

To meet this challenge, source localization approaches continue to be developed over the past 40 years and have helped to better define the spatial properties of the regions underlying EEG or MEG transients. The reconstruction of spatially extended cortical sources entails the use of distributed source models, as equivalent current dipole modelling can be misleading for large sources (Kobayashi et al., 2005). Several studies have proposed to localize extended cortical patches using beamformer (Limpiti et al., 2006; Hillebrand and Barnes, 2011), probabilistic approaches based on maximum entropy (Amblard et al., 2004; Grova et al., 2006; Chowdhury et al., 2013), subspace-based approaches (Birot et al., 2011), tensor-based techniques (Becker et al., 2015), and methods using sparsity in a transformed domain (Ding, 2009a; Zhu et al., 2014). Several of these methods showed sensitivity to source spatial extents when assessed using controlled simulations (Jerbi et al., 2002; Kincses et al., 2003; Limpiti et al., 2006; Ding, 2009a; Cheyne et al., 2010; Huiskamp et al., 2010; Birot et al., 2011; Bouet et al., 2012; Chowdhury et al., 2013; Lina et al., 2014; Becker et al., 2015) and/or when applied on real epileptic activity (Cheyne et al., 2010; Huiskamp et al., 2010; Jung et al., 2013; Zhu et al., 2013; Chowdhury et al., 2015; Grova et al., 2016; Heers et al., 2016). Among them, two approaches, developed separately by our two research teams, have been quantitatively studied for their ability to localize spatially extended sources of epileptic events, based both on simulations and real EEG and/or MEG data. The first method is a probabilistic approach based on maximum entropy, known as coherent Maximum Entropy on the Mean (cMEM), which employs a full brain parcellization model by incorporating two spatial priors: Data Driven Parcellization (DDP) and spatial coherence prior to model locally spatially smooth cortical parcels. Then, based on the state of activation of the parcels, CMEM is able to switch off some parcels while creating contrast of source intensities among other parcels. The method takes advantage of whole cortex parcellization and spatial smoothness constraint to properly recover the spatial extent of sources (Amblard et al., 2004; Grova et al., 2006, 2016; Chowdhury et al., 2013; Heers et al., 2016). The second method is a subspacebased MUSIC-like approach, known as 4-ExSo-MUSIC (4th order Extended Source Multiple Signal Classification), specifically adapted to localize extended sources by exploiting the strong synchronization of dipoles within each extended source (Birot et al., 2011). The use of $4^{\text {th }}$ order statistics in 4-ExSoMUSIC offers robustness with respect to Gaussian noise and modelling errors. In some recent studies (Chowdhury et al., 2013, 2015; Grova et al., 2016; Heers et al., 2016), it was shown that cMEM is able to recover the spatial extent and the propagation pattern of sources more accurately than Minimum Norm Estimate (MNE) (Hämäläinen and IImoniemi, 1994), standardized Low Resolution brain Electromagnetic Tomography (sLORETA) (Pascual-Marqui, 2002), dynamic Statistical Parametric Mapping (dSPM) (Dale et al., 2000) and various hierarchical Bayesian approaches (Friston et al., 2008). In other separate studies (Birot et al., 2011; Becker et al., 2014a), 4-ExSo-MUSIC estimated the spatial extent of generators of epileptic activity with more accuracy than other MUSIC-based approaches, sLORETA, 
CHAMPAGNE (Wipf et al., 2010), Minimum Current Estimate (MCE) (Uutela et al., 1999), or Mixed Norm Estimate (MxNE) (Ou et al., 2009).

In the present contribution, our first objective was to carefully compare these two methods. To do so, we used a common ground-truth well-controlled simulation environment involving complex patterns of source configurations in particular with respect to the number of sources, their spatial properties, and their level of synchronization. To this end, we used a highly realistic simulation pipeline that combines a biophysical distributed dipole source model with a computational neural mass model (Cosandier-Rimélé et al., 2008) to generate simultaneous EEG and MEG synthetic signals mimicking normal background and epileptic discharges.

Most of the studies that have compared MEG and EEG on clinical data, were acquired using less EEG electrodes than MEG sensors (Barkley and Baumgartner, 2003; Malmivuo, 2012; Lopes da Silva, 2013). These studies have demonstrated that MEG localization was more accurate. However, some simulation studies have suggested higher accuracy of EEG localization when an equivalent number of EEG and MEG channels are used (Liu et al., 2002; Song et al., 2015). The advent of dense array EEG caps and of source estimation techniques resulted in a higher spatial accuracy (Ryynanen et al., 2006; Babiloni et al., 2009; Brodbeck et al., 2011; Gavaret et al., 2015). Other studies also demonstrated that improvement in terms of localization can be attained when using a high density scalp electrodes and realistic geometry head models (Lantz and Grave de Peralta, 2003; Wang et al., 2011; Birot et al., 2014; Klamer et al., 2015). Therefore, to get the best of each modality simultaneously, the present study makes use of realistically simulated high resolution EEG (HR-EEG) and MEG data while taking into account an equivalent number of channels, realistically shaped head model and simultaneous recordings.

The outline of the paper is as follows. First, introduction of the two source localization methods (cMEM and 4-ExSo-MUSIC) is proposed, followed by the description of the realistic simulation dataset. Then, an explanation of the evaluation process and the performance criteria to assess the source localization of both the methods is given. This is followed by the description of the clinical dataset. Then the results, discussion and conclusion are provided.

\section{Methods and materials}

The goal of this study was to compare the performance of the CMEM and 4-ExSo-MUSIC algorithms on different patterns of IEDs seen in HR-EEG and MEG data, mainly to compare the ability of the methods:

- to recover the location and spatial extent of the spatially extended generators of IEDs;

- to identify the propagation patterns exhibited by IEDs;

- to handle multiple sources of IEDs with different levels of synchrony. 


\subsection{Source localization algorithms}

\subsubsection{EEG/MEG inverse solution using distributed source model}

It is commonly admitted that the EEG/MEG signals recorded at the head surface mostly reflect the activity of pyramidal cells within the cortex. These activities can be modelled by current dipoles distributed along the cortical surface, and oriented orthogonally to the cortical surface (Dale and Sereno, 1993). Using this anatomical constraint, the relationship between source amplitudes and EEG/MEG measurements can be expressed by the following linear model:

$\boldsymbol{m}(t)=\mathbf{G} \boldsymbol{j}(t)+\mathbf{e}(t)$

where $\boldsymbol{m}(t)$ is a $q$-dimensional vector of the EEG/MEG signal measured with $q=275$ MEG sensors or 257 EEG electrodes at time $t$. $\mathbf{e}(t)$ models an additive measurement noise at time $t$. $j(t)$ is an $r$-dimensional vector standing for the unknown current density along the cortical surface $(r \approx 8000$ : unknown dipolar moment amplitudes) at time $t . \mathbf{G}$ is the ( $q \times r)$ lead field matrix obtained by solving the forward problem to estimate the contribution of each dipolar source on the sensors.

To describe cMEM and 4-ExSO-MUSIC that are statistical algorithms, we introduce a random vector $\mathbf{m}$ (respectively $\mathbf{j}$ and $\mathbf{e}$ ) to model the $\tau$ recorded samples $\{\boldsymbol{m}(t)\}$ (respectively $\{\boldsymbol{j}(t)\}$ and $\{\boldsymbol{e}(t)\}$ ). According to equation $1, \mathbf{m}$ can be related to $\mathbf{j}$ and $\mathbf{e}$ as follows:

$$
m=G j+e
$$

Within the distributed dipole layer, defined as the source space $\Omega$, each dipole is characterized by its location $\rho \in \Omega$ and its current density j $(\rho)$. Thus, to describe the 4-ExSo-MUSIC algorithm (section 2.1.1.2) equation 2 is reformulated as:

$$
\mathbf{m}=\sum_{\rho \in \Omega} \boldsymbol{g}(\rho) \mathrm{j}(\rho)+\mathbf{e}
$$

where $\mathbf{j}(\rho)$ is a component of vector $\mathbf{j}$ representing the activity of a dipole at location $\rho$ and $\boldsymbol{g}(\rho)$ is a column vector of matrix $\mathbf{G}$ representing the contribution of a unitary dipole located at $\rho$ to the set of EEG/MEG sensors.

\subsubsection{1 cMEM algorithm}

In the MEM framework (Amblard et al., 2004), $\mathbf{j}$ being the $r$-dimensional continuous random variable that describes the dipole intensities, this variable has the probability distribution $d p(\mathbf{j})=p(\mathbf{j}) d \mathbf{j}$ where $\mathbf{j} \in \mathbb{R}^{r}$. 
To regularize the inverse problem, the MEM framework incorporates prior information on $\mathbf{j}$ in the form of a reference distribution $d v(\mathrm{j})$. Then, the Kullback Leibler divergence or $v$-entropy defined by:

$S_{v}(d p)=-\int_{\mathbf{j}} \log \left(\frac{d p(\mathbf{j})}{d v(\mathbf{j})}\right) d p(\mathbf{j})=-\int_{\mathbf{j}} f(\mathbf{j}) \log (f(\mathbf{j})) d v(\mathbf{j})$

measures the amount of information brought by the data with respect to the prior $d v$, where $f$ is a vdensity of $d p$ defined as, $d p(\mathbf{j})=f(\mathbf{j}) d v(\mathbf{j})$. Being a pseudo-distance between the reference distribution $d v$ and any $v$-density $d p$, this entropy is always negative.

In order to introduce a data fit constraint, let us denote $C_{m}$ as the set of probability distributions on $\mathbf{j}$ that explains the data on average:

$$
d p \in \mathrm{C}_{m}: \mathbf{m}-\left[\mathbf{G} \mid \boldsymbol{I}_{q}\right]\left[\begin{array}{l}
\mathrm{E}_{d p}[\mathbf{j}] \\
\mathbf{e}
\end{array}\right]=0
$$

where $E_{d p}[\mathbf{j}]=\int_{\mathbb{R}} \mathbf{j} d p(\mathbf{j})$ is the mathematical expectation of $\mathbf{j}$ with respect to the probability distribution $d p$ , $I_{q}$ is a $(q \times q)$ identity matrix.

Then, the MEM solution consists in selecting $d \hat{p}$ in $\mathrm{C}_{m}$ that maximizes the $v$-entropy, thus choosing the distribution fulfilling the data fit constraint that is the closest (in terms of Kullback Leibler divergence) to the reference distribution $d v$ :

$$
d \hat{p}=\operatorname{argmax}_{d p \in \mathrm{C}_{m}} S_{v}(d p)
$$

Such a regularization framework allows us to estimate the MEM solution through a non-linear optimization of a convex function within a q-dimensional space, iteratively for each time sample. This solution is unique and describes only what we "know": the prior knowledge encompassed in the reference measure $d v$, and the measurements $\{\boldsymbol{m}(t)\}$ that define the space $\mathrm{C}_{m}$ formalizing our data fit constraint (equation 5). During the MEM optimization process, the noise covariance model is estimated as a diagonal matrix with a different value for each channel; thus taking into account the noise levels of each individual channel.

The MEM estimate of the source intensities $\hat{j}$ is then computed as the mathematical expected value of the distribution $d \hat{p}$ :

$$
\hat{\mathbf{j}}=\mathrm{E}_{d \hat{p}}[\mathbf{j}]
$$

Definition of the reference distribution $d v$ : MEM relies on its inherent flexibility of introducing constraints or knowledge about the sources through the definition of the reference distribution $d v$. To do 
so, we considered brain activity to be organized into $K$ cortical parcels, each parcel showing a homogeneous activation state (Amblard et al., 2004). We used a Data Driven Parcellization (DDP) method to perform full parceling of the tessellated cortical surface into non-overlapping parcels (Lapalme et al., 2006). This spatial parcelling is driven by the Multivariate Source Pre-localization (MSP) technique (Mattout et al., 2005), which is a projection method providing a coefficient for each dipolar source characterizing its contribution to the data. For more details on the DDP method please refer to (Chowdhury et al., 2013).

Each cortical parcel $k$ is characterized by an activation state $S_{k}$, describing if the parcel is active or not. Assuming a collection of mutually independent parcels, the reference distribution $d v$ was defined as a factorization of the joint probability distribution of the $K$ parcels:

$$
d v(\mathbf{j})=\prod_{k=1}^{K}\left[\left(1-\alpha_{k}\right) \delta\left(\mathbf{j}_{k}\right)+\alpha_{k} \mathcal{N}\left(\boldsymbol{\mu}_{k}, \boldsymbol{\Sigma}_{k}\right)\left(\mathbf{j}_{k}\right)\right] d \mathbf{j}
$$

where $\alpha_{k}=\operatorname{Prob}\left(\mathrm{S}_{k}=1\right)$ is the probability of the $k^{\text {th }}$ parcel to be active. $\mathbf{j}_{k}$ denotes the random vector modelling the intensities of the $r_{k}$ sources in the $k^{\text {th }}$ parcel. $\delta$ refers to the Dirac distribution allowing to "shut down" inactive parcels when $S_{k}=0 . \mathcal{N}\left(\boldsymbol{\mu}_{k}, \boldsymbol{\Sigma}_{k}\right)$ is a Gaussian distribution modelling the intensities of the $k^{\text {th }}$ parcel when active $\left(S_{k}=1\right)$, where $\boldsymbol{\mu}_{k}$ and $\boldsymbol{\Sigma}_{k}$ represent the mean and the covariance, respectively, of the $r_{k}$ sources within the $k^{\text {th }}$ parcel. For more details on MEM regularization technique refer to Appendix A.

In the present study, we considered the variant of MEM algorithm called coherent-MEM (CMEM) implementation, as described in (Chowdhury et al., 2013). In cMEM, additional constraint of local spatial smoothness in each parcel was introduced using diffusion-based spatial priors (Friston et al., 2008) in the initialization of the source covariance of every parcel $\left(\boldsymbol{\Sigma}_{k}\right)$. This diffusion-based spatial prior is constructed using the Green's function of the adjacency or spatial connectivity matrix defined over the geodesic cortical surface (Harrison et al., 2007). The mean intensity of every parcel $\left(\boldsymbol{\mu}_{k}\right)$ was initialized to zero. The probability of activation $\left(\alpha_{k}\right)$ of each parcel $k$ was initialized at each time sample as the median of the MSP coefficients of all the $r_{k}$ sources within the $k^{\text {th }}$ parcel. The spatial neighborhood order considered for the parcels has been fixed to a scale of 4 , leading to approximately $K=200$ parcels of size $\approx 2.5 \mathrm{~cm}^{2}$. Please refer to (Chowdhury et al., 2013) for further details on the initialization of these parameters.

MEM approach is now available for users as a toolbox (namely, BEst: Brain Entropy in space and time) in the Brainstorm software (Tadel et al., 2011) and the tutorial introducing this toolbox can be downloaded ("Tutorials/TutBEst - Brainstorm," 2015). 


\subsubsection{4-ExSo-MUSIC algorithm}

4-ExSo-MUSIC is a subspace-based method which aims at extending the classical MUSIC method (Schmidt, 1986; Mosher et al., 1992) to the case of distributed sources by means of higher order statistics (Birot et al., 2011). More particularly, the sources to be localized are assumed to exhibit dipoles with highly synchronized activities (Birot et al., 2011). This property is justified by the fact that EEG/MEG signals, to be detectable on scalp, arise from the summation of highly synchronized neuronal assemblies over a spatially extended region.

Then, the EEG/MEG data can be considered as the sum of the epileptic activities arising from the subset $\Theta \subset \Omega$ and normal background activity arising from $\Omega \backslash \Theta$, such that the EEG/MEG vector is represented as follows:

$$
\mathbf{m}=\sum_{\rho \in \Theta} \boldsymbol{g}(\rho) \mathrm{j}(\rho)+\sum_{\rho \in \Omega \backslash \Theta} \boldsymbol{g}(\rho) \mathrm{j}(\rho)+\mathbf{e}=\sum_{\rho \in \Theta} \boldsymbol{g}(\rho) \mathrm{j}(\rho)+\boldsymbol{\varepsilon}
$$

where the sum over $\Theta$ models the contribution of the epileptic activity, i.e., the sources of interest while the sum over $\Omega \backslash \Theta$ models the contribution of the background activity, i.e., the sources of non-interest. In addition, let the term $\boldsymbol{\varepsilon}$ model the physiological background and the instrument noise, which can be seen as a Gaussian random vector of unknown spatial covariance and independent from the epileptic sources.

4-ExSo-MUSIC aims at estimating the spatial support $\Theta$ of the generators of epileptic activity. 4-ExSoMUSIC reformulates the spatial support $\Theta$ as the union of $B$ circular shaped disks $\Theta_{b}$, where $1 \leq b \leq B$. The search for this spatial support $\Theta$ is actually formulated within a grid containing pseudo-disks of different size over the whole cortical surface. Since the source space is a triangular mesh of the cortical surface, these pseudo-disks of different size were constructed around each vertex on the cortical surface. Each disk was composed of a maximum of 500 adjacent vertices. This set of pseudo-disks of different size for every vertex defines the so-called 4-ExSo-MUSIC grid. The model assumes that all dipolar sources belonging to one disk $\Theta_{b}$ should exhibit the same current density $j_{b}$ (and consequently the same time course). Then, equation 9 can be reformulated as follows:

$$
\begin{aligned}
& \mathbf{m}=\sum_{b=1}^{B} \mathrm{j}_{\mathrm{b}} \sum_{\rho \in \Theta_{\mathrm{b}}} \boldsymbol{g}(\rho)+\boldsymbol{\varepsilon}=\sum_{b=1}^{B} \boldsymbol{h}\left(\Theta_{\mathrm{b}}\right) \mathrm{j}_{\mathrm{b}}+\boldsymbol{\varepsilon} \\
& \mathbf{m}=\boldsymbol{H}(\Theta) \mathbf{j}_{\Theta}+\boldsymbol{\varepsilon}
\end{aligned}
$$

where the $b^{\text {th }}$ column vector of the mixing matrix $H(\Theta)$ and the $b^{\text {th }}$ component of the source random vector $\mathbf{j}_{\Theta}=\left[\mathrm{j}_{1}, \ldots, \mathrm{j}_{B}\right]^{T}$ are $\boldsymbol{h}\left(\Theta_{b}\right)$ and $\mathrm{j}_{\mathrm{b}}$, respectively.

Each set of contiguous distinct disks $\Theta_{b}$ represents a so-called Extended Source (ExSo). Moreover, 4ExSo-MUSIC relies on the assumption that the current densities of the ExSos do not follow a Gaussian 
distribution, which is an appropriate assumption for most of the signals of interest in EEG/MEG such as interictal epileptic signals (Nurujjaman et al., 2009). Therefore, the method considers the $4^{\text {th }}$ order cumulants (and more particularly their matrix form called quadricovariance) of $\mathbf{m}$ to reduce the penalizing effects of the random vector noise $\boldsymbol{\varepsilon}$ (i.e. the background cerebral activity and the instrument noise) that can be seen as a Gaussian and spatially correlated noise. The important property exploited here is that the $4^{\text {th }}$ order cumulant of a Gaussian distribution is zero, therefore one can hypothesize that 4-ExSoMUSIC would be robust with respect to the presence of background activity and instrumental noise.

Exploiting the linear model proposed in equation 10, the quadricovariance matrix, $\boldsymbol{C}_{4, \mathrm{~m}}$, of the data can be written as a function of $\Theta$ as follows:

$$
\boldsymbol{C}_{4, \mathrm{~m}}=\boldsymbol{H}(\Theta)^{\otimes 2} \boldsymbol{C}_{4, \mathrm{j}}\left[\boldsymbol{H}(\Theta)^{\otimes 2}\right]^{T}
$$

where $H(\Theta)^{\otimes 2}$ is the Kronecker product of the matrix $H(\Theta)$ by itself and $C_{4, j}$ is the quadricovariance matrix of $j_{\Theta}$. Note that there is no more contribution from the noise $\varepsilon$ since its quadricovariance is zero. Therefore, the $4^{\text {th }}$ order signal subspace of interest, defined as $\operatorname{span}\left\{H(\Theta)^{\otimes 2}\right\}$, can be directly estimated from the quadricovariance matrix $\boldsymbol{C}_{4, \mathrm{~m}} \operatorname{since} \operatorname{span}\left\{\boldsymbol{H}(\Theta)^{\otimes 2}\right\}=\operatorname{span}\left\{\boldsymbol{C}_{4, \mathrm{~m}}\right\}$.

To do so, the $4^{\text {th }}$ order signal subspace of interest is obtained from an eigenvalue decomposition of $\boldsymbol{C}_{4, \mathrm{~m}}$. 4-ExSo-MUSIC then exploits the fact that the vector $\boldsymbol{h}\left(\Theta_{b}\right)^{\otimes 2}$ must lie in the $4^{\text {th }}$ order signal subspace computed from $\boldsymbol{C}_{4, \mathrm{~m}}$.

The spatial support $\Theta=\bigcup_{1 \leq b \leq B} \Theta_{b}$ of epileptic dipoles was identified by concatenating all the candidates $\Theta_{b}$ such that $\boldsymbol{h}\left(\Theta_{b}\right)^{\otimes 2}$ belongs to the $4^{\text {th }}$ order signal subspace. To do so, the 4-ExSo-MUSIC algorithm consists in scanning the cortex with a grid of disks to estimate the candidates $\boldsymbol{h}\left(\Theta_{b}\right)^{\otimes 2}$ that minimizes the following criterion:

$\Gamma\left(\boldsymbol{E}_{4, \boldsymbol{m}}, \boldsymbol{h}\left(\Theta_{b}\right)\right)=1-\frac{\left(\left[\boldsymbol{h}\left(\Theta_{b}\right)^{\otimes 2}\right]^{\mathrm{T}} \boldsymbol{E}_{4, \mathrm{~m}} \boldsymbol{E}_{4, \mathrm{~m}}{ }^{\mathrm{T}} \boldsymbol{h}\left(\Theta_{b}\right)^{\otimes 2}\right)}{\left\|\boldsymbol{h}\left(\Theta_{b}\right)^{\otimes 2}\right\|_{2}^{2}}$

where $E_{4, \mathrm{~m}}$ is the matrix of the eigenvectors associated with the non-zero eigenvalues of $\boldsymbol{C}_{4, \mathrm{~m}}$. Providing an exhaustive search to estimate this criterion on every pseudo-disk of the 4-ExSo-MUSIC grid would not be feasible because of computational cost. Therefore, a first scan of the cortex with a coarse grid of disks, for which the size of the disks was fixed to 20 , is performed. With this first scan, a region in the cortex is selected according to the corresponding values of the metric. To define this region, the 200 lowest values of the "first-scan-metric" are identified, and the 200 corresponding disks of size 20 are concatenated. Then the original 4-ExSo-MUSIC grid (disk sizes varying from 1 to 500 dipoles) is used but only on this selected region. Finally, the relative contribution of a disk to the 4th order signal subspace (also called 
metric, as estimated through the second term $\left(\frac{\left(\left[\boldsymbol{h}\left(\Theta_{b}\right)^{\otimes 2}\right]^{\mathrm{T}} \boldsymbol{E}_{4, \mathrm{~m}} \boldsymbol{E}_{4, \mathrm{~m}}{ }^{\mathrm{T}} \boldsymbol{h}\left(\Theta_{b}\right)^{\otimes 2}\right)}{\left\|\boldsymbol{h}\left(\Theta_{b}\right)^{\otimes 2}\right\|_{2}^{2}}\right)$ of equation 12), allowed estimating the most appropriate disk size for every selected vertex. At the end of this second step, every vertex is then associated with a pseudo-disk of specific size (between 1 to 500 dipoles) and a metric value. This metric quantifies the contribution of all the dipoles of the pseudo-disk to the signal subspace.

Finally the Goodness-Of-Fit (GOF) criterion (equation 13) is used in order to select the optimal concatenation of those pseudo-disks.

$$
\mathrm{GOF}=\sqrt{\frac{\sum_{t}\left\|\boldsymbol{m}(t)-\boldsymbol{m}_{\mathrm{rec}}(t)\right\|_{2}^{2}}{\sum_{t}\|\boldsymbol{m}(t)\|_{2}^{2}}}
$$

where $\left\{\boldsymbol{m}_{\mathrm{rec}}(t)\right\}$ corresponds to the data that is reconstructed from the estimated source configuration and $\{\boldsymbol{m}(t)\}$ is the measured data matrix. The source configuration that provides the lowest GOF value is considered the best fit for the actual ExSo. This provides a binary map of the estimated source, also called the thresholded 4-ExSo-MUSIC map that is stable along time.

The time course of the estimated source configuration was obtained from a least square fit between the mixing matrix $\boldsymbol{H}(\Theta)$ for the estimated source configuration and the measured data $\{\boldsymbol{m}(t)\}$.

Similarly to all-MUSIC approaches, it is required to indicate the number of underlying ExSos a priori in the 4-ExSo-MUSIC approach. Assuming $X$ as the number of underlying sources expected a priori, the number of underlying sources considered by 4 -ExSo-MUSIC was set to $X(X+1) / 2$ when the sources were assumed to be correlated and as $X$ when the sources were assumed to be independent.

\subsection{Evaluation using realistic simulations}

In order to evaluate and compare CMEM and 4-ExSo-MUSIC within a controlled environment, realistic simulations of EEG and MEG data were generated based on information regarding the number and position of the EEG/MEG channels during a simultaneous EEG/MEG data acquisition using a 275 channel CTF-MEG system and a HR-EEG EGI system (257 electrodes). The distributed source space consisted in a mesh (8000 vertices) of the cortical surface that was obtained by segmenting the greywhite matter interface from a subject's anatomical MRI using BrainVISA-4.2.1 software ${ }^{2}$ (Mangin et al., 1995). Using the OpenMEEG (Gramfort et al., 2011) implementation in Brainstorm software (Tadel et al., 2011), we generated a 3-layer EEG Boundary Element Method (BEM) model consisting of the inner skull,

\footnotetext{
${ }^{2}$ http://www.brainvisa.info
} 
outer skull and the scalp surfaces, with corresponding conductivity values of 0.33:0.0165:0.33 S/m respectively (Ferree et al., 2000; Hoekema et al., 2003; Lai et al., 2005). For the MEG forward problem, we considered a 1-layer BEM model consisting of the inner skull surface, with brain conductivity value of $0.33 \mathrm{~S} / \mathrm{m}$.

We used a biophysical distributed dipole source model with a computational neural mass model (Wendling et al., 2000; Wendling, 2005) to generate normal background and epileptic spike-like activity. The reader may refer to (Cosandier-Rimélé et al., 2007, 2008, 2010) for detailed description of neural mass model and of the pipeline used to simulate data. In brief, we assumed a spatio-temporal source model where the neocortical pyramidal neurons were the main contributors of EEG/MEG signals. This source space was modeled by a dipolar layer distributed along the cortical surface, where each dipole corresponded to a distinct neuronal population of the neural mass model (Figure 1A). Time activities of all dipoles of the source space were generated with the neural mass model in which excitation and inhibition parameters of each neuronal population can be adjusted to obtain either epileptic spikes or background activity (Figure 1B). With an appropriate setting of coupling parameters between populations, a spatially extended generator exhibiting highly synchronized epileptic activity was constructed. The spatially extended source was made of contiguous triangles manually outlined using a mesh visualization software (Paraview, Kitware Inc., NY, US) (Figure 1C). Dipoles within the spatially extended source were associated to coupled neuronal populations, tuned to generate highly correlated epileptic activity. The remaining triangles of the mesh were then grouped into 1000 clusters. The dipoles of each cluster were then associated to a neuronal population tuned to generate normal background activity. These populations were not coupled in order to generate uncorrelated background activity. Moreover, when two spatially extended generators were simulated, spike propagation from a population $i$ to a population / was obtained by assigning the same time course to the two populations and then introducing a propagation delay drawn randomly between 16 and $20 \mathrm{~ms}$ (Figure 1B). According to the scenario considered, we varied the size, location, number of sources or the level of synchronization between neuronal populations and the time delay between populations' activities.

Once the amplitude of each elementary dipole is known the forward problem is applied in order to compute the corresponding EEG and MEG signals (Figure 1D). Then, the inverse problem consisted in inferring the sources of activity $\{j(t)\}$ based on the simulated measurement at the sensors' level $\{\boldsymbol{m}(t)\}$. Each trial consisted in the simulation of a 10s segment of EEG/MEG data (5120 time samples at $512 \mathrm{~Hz}$ ) following different simulation schemes. For the inverse problem, in the EEG gain matrix a slightly different value was used for the skull conductivity $-0.0150 \mathrm{~S} / \mathrm{m}$ instead of $0.0165 \mathrm{~S} / \mathrm{m}$.

The following scenarios have been considered. For each scenario, 20 EEG and MEG trials were simulated, each consisting of a new realization for the spiking activity and for the background activity. Consequently, each trial consisted of at least 3 or more spikes simulated within a $10 \mathrm{~s}$ window: 
1. A single source at 10 different locations: posterior bank of the central sulcus (CS), basal temporal region (BT), mesial orbito-frontal gyrus (OF), supplementary motor area (SMA), inferior parietal region $(P)$, insula, lateral orbito-frontal region, temporal pole, superior temporal region and occipital region. The source size was around $10 \mathrm{~cm}^{2}$.

2. A single source with different spatial extents at three different locations: posterior bank of the central sulcus (CS), basal temporal region (BT), inferior parietal region (P). The source area was set to $0.5 \mathrm{~cm}^{2}, 1$ $\mathrm{cm}^{2}, 2 \mathrm{~cm}^{2}, 3 \mathrm{~cm}^{2}, 4 \mathrm{~cm}^{2}, 5 \mathrm{~cm}^{2}, 7.5 \mathrm{~cm}^{2}, 10 \mathrm{~cm}^{2}, 20 \mathrm{~cm}^{2}$, and $30 \mathrm{~cm}^{2}$.

3. Two sources with synchronous activities: inferior temporal (patch 1) and parietal (patch 2) source of size $10 \mathrm{~cm}^{2}$ each. Time course of dipoles were highly synchronized in each source. In order to mimic two synchronous activities, the dipoles of the parietal source were attributed with the same temporal dynamics as the dipoles of the inferior temporal source.

4. Two Sources with propagated activity: inferior temporal (patch 1) and parietal (patch 2) source of size $10 \mathrm{~cm}^{2}$ each. Time course of dipoles were highly synchronized in each source but activities attributed to dipoles of the parietal source were temporally delayed with respect to activities attributed to the dipoles of the temporal source. The temporal delay was randomly chosen between 16 and $20 \mathrm{~ms}$ for each realization of propagating activity.

To analyze these scenarios, first the peak of the spikes were identified and marked on the simulated EEG/MEG signals. In case of very small sources where the signal-to-noise ratio of EEG and MEG signals was very low, the timing of epileptic discharge was detected from the simulated source dynamic within the generator.

In the scenarios 1 and 2, CMEM and 4-EXSo-MUSIC approaches were applied on a window of 500ms around the peak of the averaged EEG and MEG spike (generally 3 or more spikes were averaged for each trial). Therefore, we localized 20 averaged spikes for each of the 10 different source locations. For scenarios 3 and 4, a $500 \mathrm{~ms}$ window around the peak of the spike that was extracted from the $10 \mathrm{~s}$ of EEG and MEG data, then single spike localization was performed using CMEM and 4-ExSo-MUSIC. For the $4^{\text {th }}$ scenario, since CMEM provides a sample-by-sample result, we could present the source maps at the peak of the first spike and delayed spike. Since, 4-ExSo-MUSIC provides a result over a time interval, we considered a first window involving some background activity to few samples after the peak of the first spike and a second window from the onset of the second spike until few 100 samples after the second peak. For scenarios 1, 2 and 3 we indicated the number of a priori source as 1 (cf. section 2.1.1.2, $X=1$ for independent source) and for the $4^{\text {th }}$ scenario, we indicated the number of correlated sources $(X(X+1) / 2$, where $X=2$ for 2 correlated sources) a priori to the 4-ExSo-MUSIC method.

All the source localization results using CMEM have been presented after an Otsu threshold, which is obtained by taking the absolute value of the current density at the peak of the spike, normalized to its maximum activity over the whole cortical surface. This normalized map was then thresholded upon the level of background activity, through histogram analysis (Otsu, 1979). 4-ExSo-MUSIC results are 
presented as a binary map obtained after thresholding the 4-ExSo-MUSIC metric based on GOF measure (cf. section 2.1.1.2).

\subsection{Evaluation criteria}

To characterize the properties of the simulations, the following criteria were used:

(a) Signal-to-Noise Ratio (SNR) of the simulated EEG and MEG signals: this is defined as the standard deviation ratio between the signal around the peak and the background activity.

(b) Cancellation index (Ic): This index estimates the amount of overlap between signal patterns of individual sources within an active patch leading to signal cancellation (notably caused by dipolar sources oriented in opposite directions on both walls of a sulcus), as proposed by (Ahlfors et al., 2009).

$$
I c=1-\frac{\sqrt{\sum_{i=1}^{q}\left(\sum_{l \in \Phi} \boldsymbol{g}(i, I)\right)^{2}}}{\sum_{l \in \Phi} \sqrt{\sum_{i=1}^{q} \boldsymbol{g}^{2}(i, I)}}
$$

where $i$ is the index of summation over all $q$ sensors, $I$ is the index of summation over the set of dipoles in the simulated patch $\Phi \cdot \boldsymbol{g}(i, I)$ is the value of the $t^{\text {th }}$ row and $l^{\text {th }}$ column of the lead field matrix $\boldsymbol{G}$. This index ranges between 0 and $1, I c=1$ indicates full cancellation and $I c=0$ indicates no cancellation effect.

In addition, the following two validation criteria were used to compare the source localization results obtained using CMEM and 4-EXSo-MUSIC algorithm for the 4 simulation scenarios. These two criteria were used to assess the detection accuracy and the ability to recover the spatial extent of the generators. To be able to compare the source density map of CMEM with the 4-ExSo-MUSIC metric (which is a probability map), we considered the logarithm of the non-thresholded metric for 4-ExSo-MUSIC. 4-ExSoMUSIC metric provides a source map that is stable along time and CMEM provides a spatio-temporal source map. For CMEM, we therefore considered the source map at the main peak $\left(\tau_{0}\right)$ of the simulated spike for the following evaluation criteria.

(c) Area Under the Receiver Operating Characteristic (ROC) Curve, AUC: was proposed in (Grova et al., 2006) as a detection accuracy index (between 0 and 1), to assess the sensitivity of a source localization method to the spatial extent of the underlying generator. An AUC value greater than 0.8 was considered a good detection accuracy. The AUC index is assessing towards a Ground Truth the normalized energy of a source map at a specific time sample. 
AUC was estimated based on available Ground Truth, whereby, ROC curves were generated by plotting the sensitivity against the false positive detection rate for different detection thresholds, $(\beta \in[0,1])$. Normalized energy for both the estimated and the simulated current density distribution were used to quantify the amount of true positive (TP), true negative (TN), false negative (FN), and false positive (FP) for each threshold $\beta$.

However, in such context, ROC analyses are biased by the fact that a source simulation would correspond to quite more negative samples (several thousand sources) than positive ones (few hundred sources). In order to avoid such a bias, we proposed a strategy to consider the same number of active and inactive sources for ROC analysis (Grova et al., 2006). Considering the $r$ dipolar sources along the cortical surface, only few dipoles were actually active $\left(r_{a}\right)$ compared to the large number of inactive dipoles $\left(r-r_{a}\right)$. Therefore, selection of same number of inactive sources as the active sources was done by randomly selecting inactive sources among the $r-r_{a}$ sources located within the immediate spatial neighborhood of the simulated source $\left(\mathbf{A U C} \mathbf{C}_{\text {close }}\right)$ or among the local maxima of the reconstructed activity located far from the simulated source $\left(\mathbf{A U C} \mathbf{C}_{\text {far }}\right.$ ). Final $\mathbf{A U C}$ index was then computed as a mean of $\mathrm{AUC}_{\text {close }}$ and $\mathrm{AUC}_{\mathrm{far}}$ indices

In case of simulations involving a single source, the energy at the main peak of the spike was considered. For the $3^{\text {rd }}$ scenario involving two synchronous sources, the AUC index was estimated separately at the peak of each spike while removing the contribution of the vertices of the second source.

For the $4^{\text {th }}$ scenario involving two propagating sources, as explained in section 2.2, the two spikes were extracted separately to localize the sources. Therefore, it was possible to estimate AUC index at the peak of each spike while removing the contribution of the vertices of the second source.

(d) Spatial Dispersion (SD): This index, proposed in (Molins et al., 2008), measures both the spatial spread of the estimated source distribution around the true source location and the localization error between the estimated source distribution and the true source location. Let us denote by $\hat{j}$ the result of the source localization method to be evaluated. Then, $\hat{\boldsymbol{j}}\left(i, \tau_{0}\right)$ represents the amplitude of the current density distribution estimated for a dipolar source $i$ on the cortical surface at the main peak of the spike ( $\left.\tau_{0}\right)$.

To measure the SD of this result, we weight the amplitude of all the $r$ cortical sources by their minimum distances from the simulated patch using the following formula: 
$\operatorname{SD}(\hat{\boldsymbol{j}})=\sqrt{\frac{\sum_{i=1}^{r}\left(\min _{l \in \Phi}\left(\boldsymbol{D}^{2}(i, I)\right) \hat{j}^{2}\left(i, \tau_{0}\right)\right)}{\sum_{i=1}^{r} \hat{j}^{2}\left(i, \tau_{0}\right)}}$

where $\min _{l \in \Phi}(\boldsymbol{D}(i, I)$ provides the minimum Euclidean distance between the source $i$ and the sources $I$ in the simulated patch. $\Phi$ denotes the set of indices of the dipoles in the simulated patch and this minimum distance is zero when the source $i$ belongs to $\Phi$. SD values close to zero means there is no active source outside the simulated patch. Large SD values could be caused either by the presence of sources far away from the true source that are contributing to the estimated solution (spurious sources) or by the spatial spread of the reconstructed source around the true extent of the simulated patch.

(e) Shape Error (SE): this is the root mean square of the difference between the normalized simulated current density $(\boldsymbol{j t h})$ and the normalized estimated current density $(\hat{\boldsymbol{j}})$. This will assess the accuracy of the reconstructed time courses within the simulated patch. For 4-ExSo-MUSIC, all the dipoles within the estimated source have the same reconstructed time course. This time course was obtained from a least square fit between the lead field matrix for the estimated source configuration and the measured data (section 2.1.1.2). Therefore, SE was estimated as follows:

Let us consider $j$ th $(i, t)$ and $\hat{j}(i, t)$, where $i \in \Phi$ and $t$ is the time parameter.

$$
\mathrm{SE}=\sqrt{\frac{1}{\tau} \sum_{t}^{\tau}\left(\frac{m\left(j t h_{n}(t)\right)}{\max _{t}\left(\mid m\left(j t h_{n}(t)\right)\right.}-\frac{m\left(\hat{j}_{n}(t)\right)}{\max _{t}\left(\left|m\left(\hat{j}_{n}(t)\right)\right|\right)}\right)^{2}}
$$

with $m\left(j t h_{n}(t)\right)=\frac{1}{\operatorname{card}(\Phi)} \sum_{i \in \Phi} j t h_{n}(i, t)$ and $m\left(\hat{j}_{n}(t)\right)=\frac{1}{\operatorname{card}(\Phi)} \sum_{i \in \Phi} \hat{j}_{n}(i, t)$. $\operatorname{card}(\Phi)$ is the number of elements in the set $\Phi$. The subscript " $n$ " in $j t h_{n}$ or $\hat{j}_{n}$ denotes the normalization of the matrix $\hat{j}$ so that its values are between -1 and 1 , for example: $j_{n}(i, t)=|j(i, t)| / \max _{j}(\mid j(i, t)){ } \cdot \max _{t}$ is the maximum over $t$ time samples.

\subsection{Application on Clinical Datasets}

Simultaneous recording of HR 257-EEG and 275-MEG was not feasible since the HR-EEG system was available at the Neurological department of the Rennes Hospital in France while the MEG system was 
available at the Montreal Neurological Hospital in Canada. Therefore, from each center, we selected one clinical dataset from a patient with intractable focal epilepsy with available Ground Truth information such as intracranial depth EEG (iEEG) findings and MRI visible lesion (Focal Cortical Dysplasia (FCD) in both cases). Patient 1 had a 275-MEG recording and Patient 2 had a 257-EEG recording.

For patient 1, a suspected right orbitofrontal FCD was seen on MRI. This patient underwent a full presurgical evaluation at the Montreal Neurological Hospital and the acquisition was done at the MEG center of Université de Montréal on a 275 channel CTF whole-head MEG system. MEG data were bandpass filtered between 0.3 and $70 \mathrm{~Hz}$ after a DC-offset removal, and $60 \mathrm{~Hz}$ notch filter was further applied. Interictal spikes were independently visually marked in MEG traces using the DataEditor software (MISL, Vancouver, Canada) by a clinical neurophysiologist (E.K.). A total of 26 MEG spikes were found and averaged. MEG source localization was performed on the averaged spike. iEEG investigation with eight implanted electrodes (10 \pm 18 contacts; length: $2 \mathrm{~mm}$, diameter: $0.8 \mathrm{~mm} ; 1.5 \mathrm{~mm}$ apart, placed intracranially according to Talairach's stereotactic method in the right hemisphere) was further guided by the MEG source localization results within the suspected MRI lesion and revealed a focal ictal and interictal activity in the right lateral orbitofrontal region. The patient provided written informed consent for this study as approved by the Montreal Neurological Institute Research Ethics Board.

Patient 2 also presented a FCD visible on MRI in the left mesial orbitofrontal region, just above the rectus gyrus. This patient underwent a full presurgical evaluation at the Neurological department of Rennes University Hospital, including 257-channels EEG recordings (EGI, Eugene, USA). EEG data were band pass filtered between $0.3-100 \mathrm{~Hz}$. These data were reviewed for presence of IEDs by the clinical neurophysiologist (I.M.). On these scalp recordings a clear subcontinuous spike activity could be recorded interictally at the most frontopolar electrodes. A total of 85 spikes were extracted away from ocular, muscle or cardiac artifacts, and averaged. Source localization was applied on the averaged spike. During the second phase of his presurgical evaluation, the patient also underwent intracerebral iEEG recordings with 9 implanted electrodes ( $10 \pm 18$ contacts; length: $2 \mathrm{~mm}$, diameter: $0.8 \mathrm{~mm} ; 1.5 \mathrm{~mm}$ apart) placed intracranially according to Talairach's stereotactic method in the left frontal and temporal region. iEEG revealed a sub-continuous interictal activity, similar in morphology with that observed on scalp EEG, maximal in the contacts located within and around the focal lesion in the left mesial orbito-frontal region but spreads to the cingulate and to the lateral orbitofrontal region. The patient provided written informed consent for the use of his clinical data as requested by the Institutional Review Board of Rennes University Hospital.

For both clinical dataset, we presented source localization results from cMEM, 4-ExSo-MUSIC and from another standard source localization method - sLORETA (Pascual-Marqui, 2002) that is implemented in the brainstorm toolbox (Tadel et al., 2011). 
To present the 4-ExSo-MUSIC results, we provided the thresholded 4-ExSo-MUSIC map using the GOF criterion. Both CMEM and SLORETA results displayed over the cortical surface were thresholded at $30 \%$ of the maximum amplitude, following a similar approach as proposed in (Heers et al., 2016).

\section{Results}

\subsection{Evaluation using realistic simulations}

The results obtained for the first 5 source locations (CS, BT, OF, SMA, and P) of the first scenario, involving a single spatially extended source, are illustrated on Figure 2 . The results on the remaining 5 source locations have been provided as supplementary figure. The localization of single $10 \mathrm{~cm}^{2}$ sources was accurate (AUC>0.8) and was relatively similar for both 4-ExSo-MUSIC and CMEM, for most locations tested and for both EEG and MEG. EEG and MEG data simulated from these sources exhibited reasonable SNR and Ic values, although the SNR in the case of MEG signals was most of the time slightly lower than that of EEG, except for the SMA and the post-central source (tangential orientations). For these $10 \mathrm{~cm}^{2}$ single sources, 4-ExSo-MUSIC performed slightly better than cMEM for deep sources or for sources close to the interhemispheric line (OF and SMA source in Figure 2). For instance, sources localized with CMEM in the basal temporal region and in the orbitofrontal region were more lateral than their actual location, whereas for the SMA sources, CMEM recovered mainly the most superficial aspects of the generators. On the other hand, CMEM sources were slightly more accurate than 4-ExSo-MUSIC for the CS generator (for EEG mainly) and for the $P$ generator (for MEG mainly). To summarize the differences in performance of the two methods, we pooled together all the 200 source localization results (obtained from the 20 trials of the 10 source locations) and performed non-parametric Wilcoxon signed rank test to compare AUC and SD in CMEM versus 4-ExSo-MUSIC and in EEG versus MEG. We also tested whether the median of AUC distribution was significantly larger than 0.8 , since AUC value of 0.8 is usually considered as a good level of detection accuracy (Chowdhury et al., 2013). From these tests, we noticed that overall, 4-EXSo-MUSIC performed statistically significantly better than CMEM for EEG in terms of AUC $(p<0.001)$, although both methods provided overall excellent results since they were all showing median AUC significantly larger than 0.8 for both EEG and MEG $(p<0.005)$. On the other hand, AUC results for MEG did not show any statistically significant difference between the two methods $(p=0.24)$. Based on SD, cMEM exhibited SD values significantly smaller than 4-ExSo-MUSIC for EEG $(p<0.001)$ and for MEG $(p<0.005)$. In Figure 3, we provided a plot of AUC values as a function of SNR for all the 200 source localization results. We observed that the low AUC values in EEG and MEG for both CMEM and 4-ExSo-MUSIC were coming mainly from simulations when the source location was deep and the SNR was low. SE values were estimated for these single source activities (provided in Figure 2) and it was observed that the SE values were slightly lower for 4-ExSo-MUSIC than cMEM when localizing these 
single source activities, indicating a slightly better reconstruction of the temporal dynamics of the source using 4-ExSo-MUSIC.

For 3 of these locations (BT, P and CS), results with respect to the source spatial extent are provided on Figures 4 and 5. In general, the localization was assessed as accurate (i.e., median AUC value $>0.8$ ) when the source area encompassed at least $2 \mathrm{~cm}^{2}$ for cMEM and $3 \mathrm{~cm}^{2}$ for 4-ExSo-MUSIC. For EEG and for the BT and $\mathrm{P}$ source, small source areas were better retrieved with CMEM while large source areas were better estimated with 4-EXSo-MUSIC. For MEG, both CMEM and 4-ExSo-MUSIC exhibited similar performance levels, with accurate reconstructions for spatial extent larger than $2 \mathrm{~cm}^{2}$ for $\mathrm{P}$ and larger than $4 \mathrm{~cm}^{2}$ for BT. For smaller extent $\left(<3 \mathrm{~cm}^{2}\right)$ both methods exhibited large variabilities in their AUC scores (Figure 4). We noticed from the source localization results visually that both methods notably failed to recover the $0.5 \mathrm{~cm}^{2}$ BT sources from MEG data. The bad performance of 4-ExSo-MUSIC for EEG small sources was due to a wrong localization of the source while the moderately "bad" performance of cMEM for EEG large sources was due to an underestimation of the source spatial extent. Note that in MEG, median AUC values were close but below 0.8 , for both methods and for most spatial extents (except slightly better results for extents larger than $7.5 \mathrm{~cm}^{2}$ ). Actually, in most of those cases, only the most lateral portion of the BT generator was retrieved whereas most of the deepest aspects were always missed by both methods in MEG. Regarding the CS source, a different pattern was observed. CMEM clearly outperformed 4-ExSo-MUSIC for all sizes except for large sources (20 and $30 \mathrm{~cm}^{2}$ sources). This was observed both for EEG and MEG signals.

Both EEG and MEG simulated from the post central source we had similar range of SNR and Ic except for the $30 \mathrm{~cm}^{2}$ source for which MEG signal underwent higher signal cancellation and presented lower SNR than EEG (Figure 4). There was no clear relationship between SNR or Ic values and the performance of source localization: for instance, SNR values were higher for small BT sources than for the medium ones, while localization accuracy was increased. For the CS MEG source, the SNR gradually increased with the source area, while a trend towards a slight performance decrease was observed. As shown in Figure 5, SD values were consistently lower for CMEM than for 4-ExSo-MUSIC in the CS region, suggesting that 4-ExSO-MUSIC had a tendency to slightly overestimate the spatial extent, whereas CMEM more likely slightly underestimated the spatial extent. A similar behavior of the SD values was also observed for the BT and $\mathrm{P}$ regions (results not shown).

In the presence of two synchronous sources, cMEM retrieved properly both sources. For EEG signals, the spatial extent was slightly underestimated, and for MEG signals the temporal source was anterior to the actual location, while missing the main radial components of this generator. 4-ExSo-MUSIC retrieved the temporal source but largely overestimated its size, and was not able to localize the second source in the parietal region, neither from EEG nor from MEG data (Figure 6A).

When the activity of the second source was delayed by $20 \mathrm{~ms}$ to mimic a propagation pattern, the maximum of spikes was first detected on electrodes (respectively MEG sensors) facing the temporal 
region and then at electrodes (MEG sensors) facing the parietal region for both modalities. From both EEG and MEG data, CMEM localized well the temporal source at the earliest spike peak and the parietal source at the delayed peak (Figure 6B). Conversely, for EEG signal, 4-ExSo-MUSIC largely overestimated the temporal source at the first peak and the parietal one at the second peak. In that case, the estimated source included both the temporal and the parietal sources. Finally, for MEG signals, 4EXSo-MUSIC missed both sources. SE values were estimated for these propagating source activity (provided in Figure 6B) and it was observed that the SE values were lower for CMEM than for 4-ExSoMUSIC indicating a better reconstruction of the temporal dynamics of the source using CMEM for this scenario. Figure 7 displays the actual and the estimated time course of two sources with propagating activity. In this realization the temporal delay between the two sources was $20 \mathrm{~ms}$. cMEM localization was applied on the full 10 s window of the simulated EEG and MEG data. In Figure 7B and 7C, we presented the temporal dynamics of the reconstructed sources. The activity of sources reconstructed from EEG with cMEM reproduced the $20 \mathrm{~ms}$ time delay observed in the simulated source signals. Moreover, a clear difference in the amplitude between the first and second source was observed that matched the results reported on Figure 6B. Indeed, for EEG signals, the source activity reconstructed for the temporal source was stronger than for the parietal source, while for MEG the reverse was observed. 4-ExSo-MUSIC was applied on two separate windows extracted for the two spikes as explained in section 2.2. Since 4-ExSoMUSIC assumes the same temporal dynamics for all the dipoles within the extended source we were able to reconstruct a global time course for each source from EEG and MEG data (in Figure 7B and 7C). In agreement with the results reported on Figure 6B for 4-ExSo-MUSIC on EEG signal, we noticed that the reconstructed time courses were able to mimic the shape of the spiking activity for the two sources, however, the propagation delay was not well-represented. On the other hand, 4-ExSo-MUSIC on MEG signal failed to localize the two sources, which is also evident from the reconstructed time course that was not able to characterize the temporal dynamics of the simulated sources.

\subsection{Application on Clinical datasets}

Figure 8 illustrates source localization results obtained from MEG data of Patient 1. As shown in Figure $8 \mathrm{~A}$, this patient was exhibiting almost continuous large amplitude interictal spikes culminating at the level of right frontal MEG sensors. CMEM localized the spikes in the right orbitofrontal region (Figure 8B). This localization was in agreement with the area identified as the epileptogenic zone according to focal ictal and interictal activity recorded during iEEG investigation (pink outline). A small FCD was also suspected in this region, further confirmed after surgery (right orbitofrontal resection, seizure free for 12 months after the surgery). 4-ExSo-MUSIC also localized the source of MEG spikes in the orbitofrontal region. This localization was contiguous with the $\mathrm{EEEG}$ area, but more mesial (Figure $8 \mathrm{C}$ ). Results obtained with sLORETA showed a widespread area that included the iEEG outlined region but also involved the mesial 
aspect of the orbitofrontal region and the mesio-temporal region (Figure 8D). Note that additional intracerebral electrodes implanted in temporal regions did not exhibit any epileptic activity.

Figure 9 illustrates source localization results obtained from HR-EEG data of Patient 2. This patient had large amplitude interictal spikes recorded at the level of left frontal electrodes (Figure 9A). cMEM localized the spikes mainly in the left frontal pole and in the lateral orbitofrontal region (Figure 9B). This localization was more lateral and anterior to the lesion area (FCD) from where subcontinuous spikes where recorded with iEEG (pink outline). On the other hand, 4-ExSo-MUSIC localized the spikes near the anterior cingulate gyrus, above and deeper than the region identified from iEEG investigation (Figure 9C). sLORETA localized the source in the left lateral orbitofrontal region with ghost sources (Hauk et al., 2011) located far from the lesion, in temporo-mesial regions notably (Figure 9D).

\section{Discussion}

This study carefully compared the performances of two distributed source localization methods, cMEM and 4-ExSo-MUSIC, in order to assess their ability to localize the different patterns of IEDs, within a realistic simulation environment. While these two methods have been well-established previously for their sensitivity to the spatial extent of the sources of IEDs, it was important to evaluate their behavior on complex spatio-temporal patterns of IEDs such as including multiple sources, propagation patterns, and correlated sources. Both methods demonstrated the importance of factorizing multiple dipole activity within parcels of extended source in order to recover the spatial extent of the sources (Chowdhury et al., 2013; Birot et al., 2011; Heers et al., 2016; Becker et al., 2014b). However, differences between these two algorithms exist both at the level of a priori source model definition and regularization technique. While both methods assume parcellization of extended activity, 4-ExSo-MUSIC strongly relies on the nonGaussianity of the source activity while cMEM assumes a Gaussian mixture distribution of source activity in its prior model (i.e., in the definition of the reference distribution $d v$ ). However, cMEM inference is actually a Bayesian inference, where the a priori model is used to guide the solution informed by the data. Consequently, even if the Gaussian assumption is not completely fulfilled (cf. non Gaussianity of spiking activity), the inference model can still be valid and applicable. The ability of cMEM model to shutdown parcels that are not active during the regularization process permit to discard false positives in the solution space. On the other hand, the use of $4^{\text {th }}$ order statistics in 4-ExSo-MUSIC helps to eliminate the contribution of the background activity if it is assumed to be Gaussian, while allowing a more accurate reconstruction of the generators of epileptic discharges assumed to be non-Gaussian. These two respective properties of the two algorithms play an important role in providing solutions with an excellent contrast following the true extent of the source and exhibiting less distant spurious activity, unlike most conventional source localization methods such as MNE, sLORETA or dSPM (Chowdhury et al., 2013; Becker et al., 2015; Heers et al., 2016). Note that these more conventional source localization 
techniques, do recover accurately the maximum of the activity in most cases but not the spatial extent of the generators. Since 4-ExSo-MUSIC makes use of higher order statistics, the use of longer data sets to reduce the variance associated with estimating the higher order statistics is required. CMEM provides an estimate for each time sample, as long as we have a good estimate of the noise covariance matrix. In terms of computational time, for a reasonable length of the data ( $500 \mathrm{~ms}$ window), 4-ExSo-MUSIC takes only few minutes while cMEM takes around 20 minutes to process the data on a linux computer with Intel Core 2 Quad processor at a speed of $2.66 \mathrm{GHz}$ and $8 \mathrm{~GB}$ of RAM. On the other hand, 4-ExSo-MUSIC involves computation of large matrices leading to high computational complexity, thus requires high performance processors.

Table 1. Comparison of 4-ExSo-MUSIC and cMEM

\begin{tabular}{|c|c|c|}
\hline Algorithm & Advantages & Disadvantages \\
\hline 4-ExSo-MUSIC & $\begin{array}{l}\text { 1) Sensitive to spatially extended } \\
\text { sources } \\
\text { 2) Robust to Gaussian Noise } \\
\text { 3) Statistical thresholding technique } \\
\text { for the source map available }\end{array}$ & $\begin{array}{l}\text { 1) High computational complexity } \\
\text { 2) Requires a priori knowledge about } \\
\text { the number of sources } \\
\text { 3) Requires a sufficiently large number } \\
\text { of time samples to estimate the data } \\
\text { statistics } \\
\text { 4) Difficulty in localizing highly } \\
\text { correlated extended sources } \\
\text { 5) Difficulty in detecting propagation } \\
\text { patterns of sources }\end{array}$ \\
\hline cMEM & $\begin{array}{l}\text { 1) Sensitive to spatially extended } \\
\text { sources } \\
\text { 2) Provides source maps for each } \\
\text { time sample } \\
\text { 3) Does not need a priori knowledge } \\
\text { about the number of sources } \\
\text { 4) Ability to shutdown parcels that } \\
\text { are not active helps to eliminate } \\
\text { false-positives from the solution } \\
\text { space. } \\
\text { 5) Able to localize highly correlated } \\
\text { sources with their spatial extent } \\
\text { 6) Able to detect propagation } \\
\text { patterns of correlated sources }\end{array}$ & $\begin{array}{l}\text { 1) Computationally expensive for long } \\
\text { data lengths or large number of } \\
\text { dipoles in the source space, since } \\
\text { the localization requires a non-linear } \\
\text { optimization for each time sample } \\
\text { 2) Slightly overestimates the size of } \\
\text { small sources and underestimates } \\
\text { the size of large ones } \\
\text { 3) No statistical thresholding technique } \\
\text { available for source maps }\end{array}$ \\
\hline
\end{tabular}


Through this study, we showed that, in most of the considered simulation configurations, both CMEM and 4-ExSo-MUSIC were indeed sensitive to the spatial extent of the generators of IEDs. In previous studies, CMEM was evaluated in MEG on simulated sources with spatial extent ranging between $3 \mathrm{~cm}^{2}$ to $30 \mathrm{~cm}^{2}$ (Chowdhury et al., 2013) and 4-ExSo-MUSIC was evaluated in EEG on sources with spatial extent ranging between $0.5 \mathrm{~cm}^{2}$ to $20 \mathrm{~cm}^{2}$ (Birot et al., 2011). In the present study, we evaluated the two methods on sources with spatial extent ranging between $0.5 \mathrm{~cm}^{2}$ to $30 \mathrm{~cm}^{2}$. Both methods provided consistent and reliable detection accuracy for a wide range of source spatial extents (source sizes ranging from $3-20 \mathrm{~cm}^{2}$ for MEG and $3-30 \mathrm{~cm}^{2}$ for EEG). For both EEG and MEG, 4-ExSo-MUSIC localized the larger sources better than CMEM but failed to localize most small sources. This was also shown in previous studies (Birot et al., 2011; Becker et al., 2014a). For all the three sources (CS, BT, and $\mathrm{P}$ ), we noticed an overall slightly better sensitivity to the spatial extent of the larger sources on EEG data. This could be explained by the fact that these large sources lead to a higher signal cancellation in MEG than in EEG signals (Figure 4 and 5). This higher cancellation of MEG signal for the large sources can be justified by the selective sensitivity of MEG to mainly tangential activities (Ahlfors et al., 2010). 4-ExSoMUSIC performed slightly better than CMEM for deeper sources and this can be explained by the additional depth weighting applied during the 4-ExSo-MUSIC optimization step through normalization of the lead field matrix (cf. equation 12 in section 2.1.1.2).

In the presence of two correlated sources (active at the same time or after a delay), 4-ExSo-MUSIC could not separate the two correlated sources. 4-ExSo-MUSIC identified only one patch and largely overestimated its spatial extent in the two cases of two source simulations (Figure 6 and Figure 7). This can be explained by the fact that during 4-ExSo-MUSIC scan for the 2 correlated sources, the search was performed using a grid constructed for a single source (as explained in section 2.1.1.2) which then finds one patch that is largely overestimated. To improve the performance of 4-ExSo-MUSIC for the two sources scenarios, a grid search accounting for all combinations of two sources of two different spatial extent should have been considered. Such a situation would be extremely demanding in terms of computation time and would require some statistical test to assess the number of generators to consider. Note that a similar statistical approach accounting for all possible combinations of 1,2 or 3 equivalent current dipoles has been proposed in (Bénar et al., 2005) and an F-test was used to infer the number of sources to consider. Such an approach within the context of 4-ExSo-MUSIC, in order to assess the number of sources in addition to the size of each patch, is feasible in theory but not in practice because of computational load. On the other hand, cMEM was able to well-distinguish between the two sources (their location and spatial extent). The difference in the sensitivity of EEG and MEG to the two sources (patch 1 and patch 2) was also visible in the cMEM source reconstruction. While the temporal source was more sensitive to EEG than MEG, the parietal source was more sensitive to MEG than EEG. This explains the larger source amplitude for temporal source than parietal source in EEG source reconstruction using 
CMEM and vice versa in MEG source reconstruction using CMEM. Even though CMEM is a non-linear estimation of the source amplitude iteratively for each time sample, with no constraint on temporal smoothness, it was interesting to see the temporal dynamics of the sources reconstructed by cMEM exhibiting smooth time course, mimicking the dynamics of the simulated spike temporally with the two sources peaking at a delay of $20 \mathrm{~ms}$. This ability of CMEM can therefore be particularly interesting when applying methods to identify brain networks using the source time course and perform connectivity along the spike (Ana Coito, 2015). The reconstructed time course using 4-ExSo-MUSIC was reliable as long as the source was well localized. For example, in Figure 2, the SE values for the reconstructed time course using 4-ExSo-MUSIC was very low indicating that 4-ExSo-MUSIC was able to reconstruct the temporal dynamics of single source activity with high accuracy. On the other hand, for the propagating activity, 4ExSo-MUSIC found only the strongest source activity (temporal source in EEG and parietal source in MEG), which also explains why the reconstructed time course from 4-ExSo-MUSIC did not contain a clear peak for each of the two source activities at a delay of $20 \mathrm{~ms}$.

In previous studies, 4-ExSo-MUSIC on one side (Birot et al., 2011; Becker et al., 2014b) and cMEM on the other side (Chowdhury et al., 2013, 2015; Heers et al., 2016) were each compared with other standard source localization approaches such as MNE, sLORETA and their variants within the hierarchical Bayesian framework, tensor based methods and methods exploiting sparsity. In this context, and using simulations, CMEM and 4-ExSo-MUSIC proved to be most sensitive to the spatial extent of IEDs. CMEM has been extensively applied on clinical epilepsy data in several studies (Chowdhury et al., 2015; Grova et al., 2016; Heers et al., 2016; Pellegrino et al., 2016). Recently, it was also shown that the accuracy of cMEM could be further increased by exploiting the fusion of EEG and MEG (Chowdhury et al., 2015). In addition, it was also shown that CMEM is well-adapted to the study of complex spatiotemporal patterns such as seizures (Pellegrino et al., 2016). On the other hand, to date, 4-ExSo-MUSIC has only been evaluated in a few epilepsy cases (Becker et al., 2014a). Further work will have to consider larger groups of patients in whom the reliability of results can be evaluated with other investigations such as intracerebral recordings. In the meantime, other methods were proposed to remedy the problem of several correlated sources (Becker et al., 2014b; 2014a; Becker et al., 2015). Among them an approach that imposes sparsity on the variational map of the sources by characterizing the variations in the amplitude between adjacent dipoles as in (Ding, 2009b) as well as sparsity on the estimated source distribution itself has recently shown promising results (Becker et al., 2014a). Nevertheless, further validation is required in particular regarding the choice of some regularization parameters. In a recent study, Zhu et al., 2014 also showed that variation (V-) and wavelet (W-) based sparse source imaging (SSI) can be combined in order to exploit both the capability of recovering source boundaries (and thus source extents) and of compressing sources for better sparse reconstructions. This method applied on simulations as well as experimental data (language and motor responses), was able to recover the source spatial extents and to distinguish between multiple sources of activity better than the standard MNE and other variations of SSI methods. In a similar context, promising methods have been proposed 
within the Hierarchical Bayesian modelling (HBM) framework (Lucka et al., 2012; Strobbe et al., 2016). Lucka et al., 2012 proposed a fully-Bayesian inference method that was developed to localize focal sources, to correct depth localization, a well-known source of systematic error of many current density reconstruction methods, and to separate single sources in multiple-source scenarios. Strobbe et al., 2016 proposed a variational Bayesian approach called the multiple sparse volumetric priors (MSVP) to localize distributed sources and demonstrated the potential of a Bayesian approach to estimate the underlying sources of interictal activity. The MSVP approach seems inspired from a previously proposed method called $\mathrm{COH}-\mathrm{s}$, which was introduced in Chowdhury et al., 2013. $\mathrm{COH}$-s consisted in a model combining spatially extended parcels (coming from MEM-based parcellization) and smoothness constraint as covariance components within a hierarchical Bayesian model and inference based on restricted maximum likelihood estimate (Friston et al., 2006, 2008). In Chowdhury et al., 2013 we showed that MEM was more robust and reliable than $\mathrm{COH}$-s method especially in regards to the scale of the underlying parcellization. All these recently developed promising approaches should be considered in future comparative work.

One main feature of the present study was not only to compare cMEM and 4-ExSo-MUSIC together but also to quantify their respective ability to retrieve the spatial extent from EEG versus MEG signals. In previous studies, when EEG and MEG source localization were compared on clinical datasets, EEG recordings used a relatively small number of electrodes (typically <64) when compared to MEG (275) (Barkley and Baumgartner, 2003; Malmivuo, 2012; Lopes da Silva, 2013). In such a context, most of these clinical studies demonstrated that source localization from MEG signals was more accurate than EEG. Yet, in line with theoretical studies (Gevins, 1993; Srinivasan et al., 1996, 1998) showing that higher spatial resolution can be obtained with closely spaced electrodes, it has been reported that a clear improvement in terms of localization accuracy can be attained in epileptic patients when EEG is acquired with high density scalp electrodes cap, typically more than 120 electrodes (Lantz and Grave de Peralta, 2003; Holmes et al., 2008, 2010; Brodbeck et al., 2011; Yamazaki et al., 2012, 2013). This is even more true when the data are processed with realistic geometry head models (Wang et al., 2011; Birot et al., 2014) using appropriate brain-to-skull conductivity ratios (Huiskamp et al., 1999; Lantz and Grave de Peralta, 2003; Wang and Ren, 2013) or by calculating the calibrated skull conductivity from EEG/MEG data as recommended by Aydin et al., 2014.

Accordingly, some simulation studies suggested similar level of accuracy of EEG versus MEG source localization for an equivalent number of EEG and MEG channels (Liu et al., 2002; Song et al., 2015). Our results are in agreement with these results. In most simulation scenarios, EEG source localization yielded similar or better results than MEG source localization. The only exception was the case of a source in the wall of the central sulcus, which is a favorable situation for MEG in terms of orientation. Given that these are simulations, we are also dealing with the best case scenario for EEG source localization in terms of the head modelling. Therefore, the results from the simulation studies will always be a bit more in favor of EEG when compared to MEG. Recent comparisons on normal subjects, performed with a comparable 
number of channels for EEG (257) and MEG (275) in a motor and sensory task, have also confirmed this trend (Klamer et al., 2015). They demonstrated that EEG localization can reach similar or better accuracy than MEG localization when the same number of channels was considered, provided that an accurate individual EEG head model was used. Both modalities have their pros and cons based on their sensitivity. Therefore, to take advantage of the information from the two modalities, fusion of simultaneously recorded EEG-MEG data should be considered as a relevant option whenever it is possible (Aydin et al., 2014; Chowdhury et al., 2015). Aydin et al., 2014 showed on real data that a simultaneous analysis of EEG and MEG can take advantage of the fusion information, but it might also need calibrated realistic head models with appropriate conductivities especially for EEG head model. On the other hand, combined EEG-MEG data analysis using CMEM method on simulations have been shown to be robust to the modelling error such as using incorrect skull conductivities (Chowdhury et al., 2015).

In practice, simultaneous EEG and MEG recordings with similar number of EEG and MEG channels is not commonly performed. However, in the particular case of patients with epilepsy, simultaneous recordings would be very important as epileptic spikes recorded at different time might arise from slightly different regions. Therefore, to ensure that strictly the same source arrangement was at the origin of EEG and MEG signals, we conducted our study in the framework of simultaneous simulated EEG and MEG signals, taking into account an equivalent number of channels, and a realistically shaped head model. These simulations were also mandatory to quantify the performance of source localization approaches providing a "Ground Truth" that is otherwise difficult to reach when working with clinical data. In patients with epilepsy, the exact spatio-temporal organization of brain region(s) from where the IEDs arise cannot be defined with certainty. At best, it can be inferred from intracerebral recordings, that are usually not performed simultaneously with scalp EEG or MEG, except on rare occasions (Dubarry et al., 2014) and that have limited spatial sampling. The use of realistic simulation models that can mimic the epileptic generators is therefore a necessary step to validate the EEG/MEG source analysis techniques. In this realistic simulation framework, it is also necessary to avoid the so-called "inverse crime" (i.e. using the perfectly accurate geometric model for both inverse and forward problem). Therefore, to take this issue into account, we decided to modify slightly the skull conductivity values between the forward model considered for simulations and the one considered to solve the source localization inverse problem. However, we would like to point out that there are other ways to further avoid this so-called inverse crime as suggested in (Lucka et al., 2012), where they used different grids for simulation and source localization. The use of real measured background activity can also be an option, especially in low SNR conditions (Kobayashi et al., 2005; Grova et al., 2006; Chowdhury et al., 2013, 2015). In the present study we decided to consider a realistic biophysical model for simulation, allowing to simulate accurate time courses of synchronized epileptic discharges along the spatial extent of the source as proposed and evaluated in (Cosandier-Rimélé et al., 2007), instead of assuming a uniform simulation profile. 
The organization of neural activity in the brain is very complex and the relationship between underlying generators and recorded electro-magnetic signals is difficult to model. The simple static simulation models commonly used are a single dipole (Fuchs et al., 1998; Pascual-Marqui, 2002) or patch of dipoles with uniform activity (Liu et al., 2002; Trujillo-Barreto et al., 2004; Grova et al., 2006; Chowdhury et al., 2013). This patch with uniform activity can be extended to simulate different spatial extents of the source but does not model accurately the individual temporal course of dipoles constituting the patch. The fact that the patch extends in all directions with uniform intensity is not realistic and can be a drawback for MEG and EEG. This bias is expected to have more influence on MEG. Indeed, since MEG is selectively sensitive to sulcal sources when, for instance, a patch including two opposing walls of a sulcus would lead to an increased amount of signal cancellation when exactly the same time course is assigned along the patch (Ahlfors et al., 2009). Therefore, more realistic simulation models are required for modeling epileptic activity. Amongst the most realistic modeling approaches, neuronal computational models have been proposed. Biologically inspired neuronal mass models (Wilson and Cowan, 1972; Lopes da Silva et al., 1976; Traub, 1979) have been widely used to study and model brain activity. In this context realistic microscopic and macroscopic models have been proposed and evaluated. The macroscopic models, also called "lumped" models, are more adapted to model spatially extended activities by coupling multiple populations of cells. These biologically inspired neuron models can provide accurate description of the temporal activity of epileptic events (Wendling et al., 2000; Wendling, 2005). To describe the spatial features of the extended source, dipolar layer distributed along the cortical surface can be considered where each dipole corresponds to a distinct neuronal population. With this spatio-temporal model, that allows to vary the geometry of the source while keeping a realistic description of the temporal source, signals resembling real epileptic events can be generated at the level of intracerebral contact, EEG electrodes (Cosandier-Rimélé et al., 2007, 2008, 2010) or MEG sensors (Badier et al., 2007). This model provided the ideal framework to evaluate CMEM and 4-ExSo-MUSIC using a common simulation environment for EEG and MEG sources in epilepsy. In particular, in such a realistic framework, we could assess in detail the ability of source localization methods to reconstruct precisely the time course of sources in scenarios simulating propagation patterns.

In order to test the accuracy of CMEM and 4-ExSo-MUSIC on clinical EEG and MEG data, we chose patients for which the existence of a very focal lesion allows for making strong hypothesis on the origin of epileptic interictal activity. The other interest of choosing patients with focal cortical dysplasia was that sources of interictal activity are meant to stay relatively stable in space (Bast et al., 2004). Therefore averaging of spikes could be performed with a minimal risk of mixing activities arising from different spatial configurations, and therefore without increasing the spatial smoothness of the source estimates. This is not always the case however, therefore, averaging should be considered cautiously, and the subaveraging technique proposed by (Aydin et al., 2015) can be considered for activities whose spatial position changes in a dynamic manner within the epileptogenic tissue. In patient 1, both cMEM and 4ExSo-MUSIC were able to retrieve a source of IEDs that was in concordance with the iEEG findings and 
the lesion suggested on MRI, in line with our quantitative MEG/iEEG comparison provided in Figure 2 of (Grova et al., 2016). In this case, cMEM provided better sensitivity to the spatial extent than both 4-ExSoMUSIC and SLORETA. For patient 2, the result of both methods was slightly away from the small mesial FCD. This result was too lateral for CMEM, and too deep for 4-ExSo-MUSIC. It is interesting to notice however that these two "mislocations" corresponded to brain areas where epileptic spikes were spreading. In this regard, these localizations were not fully consistent with depth recordings but could not be considered as misleading either. In both the clinical cases, sLORETA was able to localize the sources but retrieved also a widespread area, with spurious sources in regions that were not shown to be involved during intracerebral interictal spikes. CMEM and 4-ExSo-MUSIC are therefore good candidates to be used in clinical practice of source localization of epileptic discharges and to guide iEEG implantation, although further validation on more clinical datasets is required but was out of the scope of the present study.

\section{Conclusion}

In this paper, we quantitatively assessed the behavior of two source localization methods, CMEM and 4ExSo-MUSIC, when localizing complex spatio-temporal patterns of IEDs using simulations of EEG and MEG data generated from biophysical computational neural mass model. While both the methods were studied separately and well-established for their sensitivity to the spatial extent of the generators, our goal was to compare the two methods together on a common ground of well-controlled realistic simulations while taking into account simultaneously recorded HR-EEG and MEG data. Overall, our results demonstrate the eligibility of both 4-ExSo-MUSIC and cMEM for application on clinical data due to their high sensitivity to the location and spatial extent of the generators of epileptic discharges in EEG and MEG. The superior performance of 4-ExSo-MUSIC when dealing with single source of large signal-tonoise ratio, and superior performance of CMEM when dealing with complex spatio-temporal propagation patterns suggests that the two methods provides interesting complementarities that should be taken into account when localizing clinical data.

\section{Acknowledgements}

This work was supported by SAVOY FOUNDATION, NSERC Discovery grant, CIHR (MOP-93614 and MOP-133619), Centres of Excellence for Commercialization of Research (CECR) and the American Epilepsy Society award. The acquisition of the HR-EEG system was supported by a grant from the French Foundation for Research on Epilepsy (FFRE).

\section{Appendix A}




\section{MEM regularization}

In the MEM optimization (equation 4), the $v$-entropy is strictly a convex function that needs to be maximized under constraints, which is equivalent to maximizing an unconstrained strictly concave Lagrangian function. In the Lagrangian function, the Lagrangian parameters $\kappa$ and $\lambda$ are used to add constraints to the objective function $S_{V}(d p)$, as follows:

$$
\begin{gathered}
L(d p, \kappa, \lambda)=-S_{v}(d p)+\lambda^{T}\left(\mathbf{m}-\mathbf{G E}_{d p}[\mathbf{j}]\right)+\kappa\left(1-\int d p(\mathbf{j})\right) \\
\text { i.e., } L(d p, \kappa, \lambda)=\int f(\mathbf{j}) \log f(\mathbf{j}) d v(\mathbf{j})+\lambda^{T}\left(\mathbf{m}-\mathbf{G E}_{d p}[\mathbf{j}]\right)+\kappa\left(1-\int d p(\mathbf{j})\right)
\end{gathered}
$$

where the first term is the v-entropy, second term is the data goodness of fit, and the last term expresses the constraint that $d p(\mathbf{j})$ must be a probability distribution.

Therefore the MEM formalism consists in a duality principle where the primal solutions (equation 6 and 7 ) are given as a function of the Lagrange multipliers (equation (A2) and (A5), respectively).

Therefore, the optimal solution $(d \hat{p}, \tilde{\kappa}, \tilde{\lambda})$ of this optimization problem calculated via the Lagrangian formalism, i.e., $\operatorname{argmin}_{d p, \kappa, \lambda} L(d p, \kappa, \lambda)$, provides:

$$
d \hat{p}(\mathbf{j})=\frac{e^{\tilde{\lambda}^{\top} G \mathbf{j}}}{Z(\tilde{\lambda})} d v(\mathbf{j})
$$

where $\tilde{\lambda}$ is the maximum of the non-linear optimization of a convex function $D(\lambda)$ in a $q$-dimensional space, thus accepting a unique solution. In practice, the optimization problem depends only on the parameter $\lambda$ which is the same dimension as the number of sensors $(q)$.

$$
\tilde{\lambda}=\operatorname{argmax}_{\lambda} D(\lambda) \text {, where } D(\lambda)=\lambda^{T} \mathbf{m}-F_{v}\left(\boldsymbol{G}^{T} \lambda\right)-\frac{1}{2} \lambda^{T} \boldsymbol{\Sigma}_{e} \boldsymbol{\Sigma}_{e}^{T} \lambda
$$

and the normalizing constant in equation (A2), $Z(\tilde{\lambda})=e^{F_{v}\left(G^{T} \tilde{\lambda}\right)}$ is the partition function and $F_{v}$ is the free energy associated with the reference distribution $d v$, defined as the log of the partition function.

$$
F_{v}(\boldsymbol{\xi})=\log \int e^{\xi^{T} \mathbf{j}} d v(\mathbf{j}) \text { with } \boldsymbol{\xi}=\boldsymbol{G}^{T} \tilde{\lambda}
$$

and $\boldsymbol{\Sigma}_{e}$ is the noise covariance matrix for $\mathbf{e}$ in equation 1.

It can then be shown that the primal solution in equation 7 giving the MEM estimate of the sources' intensities $\mathbf{j}$ could then be related to the dual solution as the gradient of the free energy $F_{v}$ :

$$
\hat{\mathbf{j}}_{M E M}=\left.\nabla F_{v}(\xi)\right|_{\xi=G^{\top} \tilde{\lambda}}
$$


When applied to the reference distribution introduced in equation 8, the MEM estimate of the sources in each parcel $k$ can be found to be:

$$
\hat{\mathbf{j}}_{M E M}^{k}=\hat{\alpha}_{k}\left[\boldsymbol{\mu}_{k}+\boldsymbol{\Sigma}_{k} \boldsymbol{G}_{k}^{T} \tilde{\lambda}\right]
$$

$$
\text { where } \hat{\alpha}_{k}=\frac{\alpha_{k}}{\alpha_{k}+\left(1-\alpha_{k}\right) \exp \left(-F_{v, k}\left(\boldsymbol{G}_{k}^{T} \tilde{\lambda}\right)\right)}
$$

where $F_{v, k}$ is the free energy corresponding to the $k^{\text {th }}$ parcel when active (i.e., $S_{k}=1$ ), given by:

$$
F_{v, k}\left(\boldsymbol{G}_{k}^{T} \tilde{\lambda}\right)=\boldsymbol{\mu}_{k}^{T} \boldsymbol{G}_{k}^{T} \tilde{\lambda}+\frac{1}{2} \tilde{\lambda}^{T} \boldsymbol{G}_{k} \boldsymbol{\Sigma}_{k} \boldsymbol{G}_{k}^{T} \tilde{\lambda}
$$

and $\boldsymbol{G}_{k}$ is the $\left(q \times r_{k}\right)$ submatrix of $\boldsymbol{G}$ for the $k^{\text {th }}$ parcel.

\section{References:}

Ahlfors, S.P., Han, J., Belliveau, J.W., Hämäläinen, M.S., 2010. Sensitivity of MEG and EEG to Source Orientation. Brain Topogr. 23 (3), 227-232.

Ahlfors, S.P., Han, J., Lin, F.-H., Witzel, T., Belliveau, J.W., Hämäläinen, M.S., Halgren, E., 2009. Cancellation of EEG and MEG signals generated by extended and distributed sources. Hum. Brain Mapp. 31 (1), 140-149.

Amblard, C., Lapalme, E., Lina, J.-M., 2004. Biomagnetic Source Detection by Maximum Entropy and Graphical Models. IEEE Trans. Biomed. Eng. 51 (3), 427-442.

Ana Coito, G.P., 2015. Dynamic directed interictal connectivity in left and right temporal lobe epilepsy. Epilepsia 56 (2).

Aydin, Ü., Vorwerk, J., Dümpelmann, M., Küpper, P., Kugel, H., Heers, M., Wellmer, J., Kellinghaus, C., Haueisen, J., Rampp, S., Stefan, H., Wolters, C.H., 2015. Combined EEG/MEG Can Outperform Single Modality EEG or MEG Source Reconstruction in Presurgical Epilepsy Diagnosis. PLoS ONE 10 (3).

Aydin, Ü., Vorwerk, J., Küpper, P., Heers, M., Kugel, H., Galka, A., Hamid, L., Wellmer, J., Kellinghaus, C., Rampp, S., Wolters, C.H., 2014. Combining EEG and MEG for the reconstruction of epileptic activity using a calibrated realistic volume conductor model. PloS One 9 (3), e93154.

Babiloni, C., Pizzella, V., Gratta, C.D., Ferretti, A., Romani, G.L., 2009. Fundamentals of electroencefalography, magnetoencefalography, and functional magnetic resonance imaging. Int. Rev. Neurobiol. 86, 67-80.

Badier, J.-M., Cosandier-Rimélé, D., Bénar, C.G., Schwartz, D., Chauvel, P., Wendling, F., 2007. Realistic synthetic background neuronal activity for the analysis of MEG probe configurations. Conf. Proc. Annu. Int. Conf. IEEE Eng. Med. Biol. Soc. IEEE Eng. Med. Biol. Soc. Conf. 2007, 2460-2463.

Barkley, G.L., Baumgartner, C., 2003. MEG and EEG in epilepsy. J. Clin. Neurophysiol. Off. Publ. Am. Electroencephalogr. Soc. 20 (3), 163-178.

Bast, T., Oezkan, O., Rona, S., Stippich, C., Seitz, A., Rupp, A., Fauser, S., Zentner, J., Rating, D., Scherg, M., 2004. EEG and MEG Source Analysis of Single and Averaged Interictal Spikes Reveals Intrinsic Epileptogenicity in Focal Cortical Dysplasia. Epilepsia 45 (6), 621-631.

Becker, H., Albera, L., Comon, P., Gribonval, R., Merlet, I., 2014. Fast, variation-based methods for the analysis of extended brain sources, in: Signal Processing Conference (EUSIPCO), 2014 
Proceedings of the 22nd European. Presented at the Signal Processing Conference (EUSIPCO), 2014 Proceedings of the 22nd European, pp. 41-45.

Becker, H., Albera, L., Comon, P., Gribonval, R., Wendling, F., Merlet, I., 2015. Brain-Source Imaging: From sparse to tensor models. IEEE Signal Process. Mag. 32 (6), 100-112.

Becker, H., Albera, L., Comon, P., Haardt, M., Birot, G., Wendling, F., Gavaret, M., Bénar, C.G., Merlet, I., 2014a. EEG extended source localization: Tensor-based vs. conventional methods. Neurolmage $96,143-157$.

Becker, H., Albera, L., Comon, P., Haardt, M., Birot, G., Wendling, F., Gavaret, M., Bénar, C.G., Merlet, I., 2014b. EEG extended source localization: Tensor-based vs. conventional methods. Neurolmage 96, $143-157$.

Bénar, C.G., Gunn, R.N., Grova, C., Champagne, B., Gotman, J., 2005. Statistical maps for EEG dipolar source localization. IEEE Trans. Biomed. Eng. 52 (3), 401-413.

Birot, G., Albera, L., Wendling, F., Merlet, I., 2011. Localization of extended brain sources from EEG/MEG: the ExSo-MUSIC approach. Neurolmage 56 (1), 102-113.

Birot, G., Spinelli, L., Vulliémoz, S., Mégevand, P., Brunet, D., Seeck, M., Michel, C.M., 2014. Head model and electrical source imaging: A study of 38 epileptic patients. Neurolmage Clin. 5, 77-83.

Bouet, R., Jung, J., Delpuech, C., Ryvlin, P., Isnard, J., Guenot, M., Bertrand, O., Mauguière, F., 2012. Towards source volume estimation of interictal spikes in focal epilepsy using magnetoencephalography. Neurolmage 59 (4), 3955-3966.

Brodbeck, V., Spinelli, L., Lascano, A.M., Wissmeier, M., Vargas, M.-I., Vulliemoz, S., Pollo, C., Schaller, K., Michel, C.M., Seeck, M., 2011. Electroencephalographic source imaging: a prospective study of 152 operated epileptic patients. Brain 134 (10), 2887-2897.

Cheyne, D., Lerch, J., Mohamed, I., Ferrari, P., Lalancette, M., Pang, E., 2010. Realistic models of spatially extended cortical activity in MEG. Front. Neurosci. 4.

Chowdhury, R.A., Lina, J.M., Kobayashi, E., Grova, C., 2013. MEG Source Localization of Spatially Extended Generators of Epileptic Activity: Comparing Entropic and Hierarchical Bayesian Approaches. PLoS ONE 8 (2), e55969.

Chowdhury, R.A., Zerouali, Y., Hedrich, T., Heers, M., Kobayashi, E., Lina, J.-M., Grova, C., 2015. MEGEEG Information Fusion and Electromagnetic Source Imaging: From Theory to Clinical Application in Epilepsy. Brain Topogr. 1-28.

Cooper, R., Winter, A.L., Crow, H.J., Walter, W.G., 1965. Comparison of subcortical, cortical and scalp activity using chronically indwelling electrodes in man. Electroencephalogr. Clin. Neurophysiol. 18, 217-228.

Cosandier-Rimélé, D., Badier, J.-M., Chauvel, P., Wendling, F., 2007. A physiologically plausible spatiotemporal model for EEG signals recorded with intracerebral electrodes in human partial epilepsy. IEEE Trans. Biomed. Eng. 54 (3), 380-388.

Cosandier-Rimélé, D., Merlet, I., Badier, J.M., Chauvel, P., Wendling, F., 2008. The neuronal sources of EEG: Modeling of simultaneous scalp and intracerebral recordings in epilepsy. Neurolmage 42 (1), 135-146.

Cosandier-Rimélé, D., Merlet, I., Bartolomei, F., Badier, J.-M., Wendling, F., 2010. Computational modeling of epileptic activity: from cortical sources to EEG signals. J. Clin. Neurophysiol. Off. Publ. Am. Electroencephalogr. Soc. 27 (6), 465-470.

Dale, A., Sereno, M., 1993. Improved localization of cortical activity by Combining EEG and MEG with MRI Cortical Surface Reconstruction.

Dale, A.M., Liu, A.K., Fischl, B.R., Buckner, R.L., Belliveau, J.W., Lewine, J.D., Halgren, E., 2000. Dynamic statistical parametric mapping: combining fMRI and MEG for high-resolution imaging of cortical activity. Neuron 26 (1), 55-67.

Ding, 2009a. L1-norm and L2-norm neuroimaging methods in reconstructing extended cortical sources from EEG, in: Engineering in Medicine and Biology Society, 2009. EMBC 2009. Annual International Conference of the IEEE. Presented at the Engineering in Medicine and Biology Society, 2009. EMBC 2009. Annual International Conference of the IEEE, pp. 1922-1925.

Ding, L., 2009b. Reconstructing cortical current density by exploring sparseness in the transform domain. Phys. Med. Biol. 54 (9), 2683-2697.

Dubarry, A.-S., Badier, J.-M., Trébuchon-Da Fonseca, A., Gavaret, M., Carron, R., Bartolomei, F., Liégeois-Chauvel, C., Régis, J., Chauvel, P., Alario, F.-X., Bénar, C.-G., 2014. Simultaneous 
recording of MEG, EEG and intracerebral EEG during visual stimulation: from feasibility to singletrial analysis. Neurolmage 99, 548-558.

Ebersole, J.S., 1997. Defining epileptogenic foci: past, present, future. J. Clin. Neurophysiol. Off. Publ. Am. Electroencephalogr. Soc. 14 (6), 470-483.

Ferree, T.C., Eriksen, K.J., Tucker, D.M., 2000. Regional head tissue conductivity estimation for improved EEG analysis. IEEE Trans. Biomed. Eng. 47 (12), 1584-1592.

Friston, K., Harrison, L., Daunizeau, J., Kiebel, S., Phillips, C., Trujillo-Barreto, N., Henson, R., Flandin, G., Mattout, J., 2008. Multiple sparse priors for the M/EEG inverse problem. Neurolmage 39 (3), 1104-1120.

Friston, K., Mattout, J., Trujillo-Barreto, N., Ashburner, J., Penny, W., 2006. Variational free energy and the Laplace approximation. Neurolmage 34 (1), 220-234.

Fuchs, M., Wagner, M., Wischmann, H.-A., Köhler, T., Theißen, A., Drenckhahn, R., Buchner, H., 1998. Improving source reconstructions by combining bioelectric and biomagnetic data. Electroencephalogr. Clin. Neurophysiol. 107 (2), 93-111.

Gavaret, M., Maillard, L., Jung, J., 2015. High-resolution EEG (HR-EEG) and magnetoencephalography (MEG). Neurophysiol. Clin. Neurophysiol. 45 (1), 105-111.

Gevins, A., 1993. High resolution EEG. Brain Topogr. 5 (4), 321-325.

Gramfort, A., Papadopoulo, T., Olivi, E., Clerc, M., 2011. Forward Field Computation with OpenMEEG. Comput. Intell. Neurosci. 2011, 1-13.

Grova, C., Aiguabella, M., Zelmann, R., Lina, J.-M., Hall, J.A., Kobayashi, E., 2016. Intracranial EEG potentials estimated from MEG sources: A new approach to correlate MEG and IEEG data in epilepsy: Correlation between MEG Sources and iEEG Data for Epileptic Spikes. Hum. Brain Mapp.

Grova, C., Daunizeau, J., Lina, J.M., Benar, C.G., Benali, H., Gotman, J., 2006. Evaluation of EEG localization methods using realistic simulations of interictal spikes. Neuroimage 29 (3), 734-753.

Hämäläinen, M.S., IImoniemi, R.J., 1994. Interpreting magnetic fields of the brain: minimum norm estimates. Med. Biol. Eng. Comput. 32 (1), 35-42.

Hari, R., 1990. The neuromagnetic method in the study of the human auditory cortex. ResearchGate 6.

Harrison, L.M., Penny, W., Ashburner, J., Trujillo-Barreto, N., Friston, K.J., 2007. Diffusion-based spatial priors for imaging. Neurolmage 38 (4), 677-695.

Hauk, O., Wakeman, D.G., Henson, R., 2011. Comparison of noise-normalized minimum norm estimates for MEG analysis using multiple resolution metrics. Neurolmage 54 (3), 1966-1974.

Heers, M., Chowdhury, R.A., Hedrich, T., Dubeau, F., Hall, J.A., Lina, J.-M., Grova, C., Kobayashi, E., 2016. Localization Accuracy of Distributed Inverse Solutions for Electric and Magnetic Source Imaging of Interictal Epileptic Discharges in Patients with Focal Epilepsy. Brain Topogr. 29 (1), 162-181.

Hillebrand, A., Barnes, G.R., 2011. Practical constraints on estimation of source extent with MEG beamformers. Neurolmage 54 (4), 2732-2740.

Hoekema, R., Wieneke, G.H., Leijten, F.S.S., van Veelen, C.W.M., van Rijen, P.C., Huiskamp, G.J.M., Ansems, J., van Huffelen, A.C., 2003. Measurement of the conductivity of skull, temporarily removed during epilepsy surgery. Brain Topogr. 16 (1), 29-38.

Holmes, M.D., Brown, M., Tucker, D.M., Saneto, R.P., Miller, K.J., Wig, G.S., Ojemann, J.G., 2008 Localization of extratemporal seizure with noninvasive dense-array EEG. Comparison with intracranial recordings. Pediatr. Neurosurg. 44 (6), 474-479.

Holmes, M.D., Tucker, D.M., Quiring, J.M., Hakimian, S., Miller, J.W., Ojemann, J.G., 2010. Comparing noninvasive dense array and intracranial electroencephalography for localization of seizures. Neurosurgery 66 (2), 354-362.

Huiskamp, G., Agirre-Arrizubieta, Z., Leijten, F., 2010. Regional Differences in the Sensitivity of MEG for Interictal Spikes in Epilepsy. Brain Topogr. 23 (2), 159-164.

Huiskamp, G., Vroeijenstijn, M., van Dijk, R., Wieneke, G., van Huffelen, A.C., 1999. The need for correct realistic geometry in the inverse EEG problem. IEEE Trans. Biomed. Eng. 46 (11), 1281-1287.

Jerbi, K., Mosher, J.C., Baillet, S., Leahy, R.M., 2002. On MEG forward modelling using multipolar expansions. Phys. Med. Biol. 47 (4), 523-555.

Jung, J., Bouet, R., Delpuech, C., Ryvlin, P., Isnard, J., Guenot, M., Bertrand, O., Hammers, A., Mauguière, F., 2013. The value of magnetoencephalography for seizure-onset zone localization 
in magnetic resonance imaging-negative partial epilepsy. Brain J. Neurol. 136 (Pt 10), 31763186.

Kincses, W.E., Braun, C., Kaiser, S., Grodd, W., Ackermann, H., Mathiak, K., 2003. Reconstruction of extended cortical sources for EEG and MEG based on a Monte-Carlo-Markov-chain estimator. Hum. Brain Mapp. 18 (2), 100-110.

Klamer, S., Elshahabi, A., Lerche, H., Braun, C., Erb, M., Scheffler, K., Focke, N.K., 2015. Differences between MEG and high-density EEG source localizations using a distributed source model in comparison to fMRI. Brain Topogr. 28 (1), 87-94.

Kobayashi, K., Yoshinaga, H., Ohtsuka, Y., Gotman, J., 2005. Dipole modeling of epileptic spikes can be accurate or misleading. Epilepsia 46 (3), 397-408.

Lai, Y., van Drongelen, W., Ding, L., Hecox, K.E., Towle, V.L., Frim, D.M., He, B., 2005. Estimation of in vivo human brain-to-skull conductivity ratio from simultaneous extra- and intra-cranial electrical potential recordings. Clin. Neurophysiol. Off. J. Int. Fed. Clin. Neurophysiol. 116 (2), 456-465.

Lantz, G., Grave de Peralta, R., 2003. Epileptic source localization with high density EEG: how many electrodes are needed? Clin. Neurophysiol. Off. J. Int. Fed. Clin. Neurophysiol. 114 (1), 63-9.

Lapalme, E., Lina, J.M., Mattout, J., 2006. Data-driven parceling and entropic inference in MEG. Neurolmage 30 (1), 160-171.

Limpiti, T., Van Veen, B.D., Wakai, R.T., 2006. Cortical patch basis model for spatially extended neural activity. IEEE Trans. Biomed. Eng. 53 (9), 1740-1754.

Lina, J.M., Chowdhury, R., Lemay, E., Kobayashi, E., Grova, C., 2014. Wavelet-Based Localization of Oscillatory Sources From Magnetoencephalography Data. IEEE Trans. Biomed. Eng. 61 (8), 2350-2364.

Liu, A.K., Dale, A.M., Belliveau, J.W., 2002. Monte Carlo simulation studies of EEG and MEG localization accuracy. Hum. Brain Mapp. 16 (1), 47-62.

Lopes da Silva, F., 2013. EEG and MEG: relevance to neuroscience. Neuron 80 (5), 1112-1128.

Lopes da Silva, F.H., van Rotterdam, A., Barts, P., van Heusden, E., Burr, W., 1976. Models of neuronal populations: the basic mechanisms of rhythmicity. Prog. Brain Res. 45, 281-308.

Lucka, F., Pursiainen, S., Burger, M., Wolters, C.H., 2012. Hierarchical Bayesian inference for the EEG inverse problem using realistic FE head models: depth localization and source separation for focal primary currents. Neurolmage 61 (4), 1364-1382.

Malmivuo, J., 2012. Comparison of the properties of EEG and MEG in detecting the electric activity of the brain. Brain Topogr. 25 (1), 1-19.

Mangin, J.-F., Frouin, V., Bloch, I., Régis, J., López-Krahe, J., 1995. From 3D magnetic resonance images to structural representations of the cortex topography using topology preserving deformations. J Math Imaging Vis 5 (4), 297-318.

Mattout, J., Pélégrini-Issac, M., Garnero, L., Benali, H., 2005. Multivariate source prelocalization (MSP): Use of functionally informed basis functions for better conditioning the MEG inverse problem. Neurolmage 26 (2), 356-373.

Merlet, I., Gotman, J., 1999. Reliability of dipole models of epileptic spikes. Clin. Neurophysiol. 110 (6), 1013-1028.

Mikuni, N., Nagamine, T., Ikeda, A., Terada, K., Taki, W., Kimura, J., Kikuchi, H., Shibasaki, H., 1997. Simultaneous Recording of Epileptiform Discharges by MEG and Subdural Electrodes in Temporal Lobe Epilepsy. Neuroimage 5 (4), 298-306.

Molins, A., Stufflebeam, S.M., Brown, E.N., Hämäläinen, M.S., 2008. Quantification of the benefit from integrating MEG and EEG data in minimum I2-norm estimation. Neurolmage 42 (3), 1069-1077.

Mosher, J.C., Lewis, P.S., Leahy, R.M., 1992. Multiple dipole modeling and localization from spatiotemporal MEG data. IEEE Trans. Biomed. Eng. 39 (6), 541-557.

Nurujjaman, M., Narayanan, R., lyengar, A.S., 2009. Comparative study of nonlinear properties of EEG signals of normal persons and epileptic patients. Nonlinear Biomed. Phys. 3, 6.

Oishi, M., Otsubo, H., Kameyama, S., Morota, N., Masuda, H., Kitayama, M., Tanaka, R., 2002. Epileptic spikes: magnetoencephalography versus simultaneous electrocorticography. Epilepsia 43 (11), 1390-1395.

Otsu, 1979. A Threshold Selection Method from Gray-Level Histograms. IEEE Trans. Syst. Man Cybern. $9(1), 62-66$.

Ou, W., Hämäläinen, M.S., Golland, P., 2009. A distributed spatio-temporal EEG/MEG inverse solver. Neurolmage 44 (3), 932-946. 
Pascual-Marqui, R.D., 2002. Standardized low-resolution brain electromagnetic tomography (sLORETA): Technical details. Methods Find. Exp. Clin. Pharmacol. 24 (SUPPL. D), 5-12.

Pellegrino, G., Hedrich, T., Chowdhury, R., Hall, J.A., Lina, J.-M., Dubeau, F., Kobayashi, E., Grova, C., 2016. Source localization of the seizure onset zone from ictal EEG/MEG data. Hum. Brain Mapp.

Ryynanen, O.R.M., Hyttinen, J.A.K., Malmivuo, J.A., 2006. Effect of measurement noise and electrode density on the spatial resolution of cortical potential distribution with different resistivity values for the skull. IEEE Trans. Biomed. Eng. 53 (9), 1851-1858.

Schmidt, R.O., 1986. Multiple emitter location and signal parameter estimation. IEEE Trans. Antennas Propag. 34 (3), 276-280.

Song, J., Davey, C., Poulsen, C., Luu, P., Turovets, S., Anderson, E., Li, K., Tucker, D., 2015. EEG source localization: Sensor density and head surface coverage. J. Neurosci. Methods 256, 9-21.

Srinivasan, R., Nunez, P.L., Tucker, D.M., Silberstein, R.B., Cadusch, P.J., 1996. Spatial sampling and filtering of EEG with spline Laplacians to estimate cortical potentials. Brain Topogr. 8 (4), 355366.

Srinivasan, R., Tucker, D.M., Murias, M., 1998. Estimating the spatial Nyquist of the human EEG. Behav. Res. Methods Instrum. Comput. 30 (1), 8-19.

Strobbe, G., Carrette, E., López, J.D., Montes Restrepo, V., Van Roost, D., Meurs, A., Vonck, K., Boon, P., Vandenberghe, S., van Mierlo, P., 2016. Electrical source imaging of interictal spikes using multiple sparse volumetric priors for presurgical epileptogenic focus localization. Neurolmage Clin. 11, 252-263.

Tadel, F., Baillet, S., Mosher, J.C., Pantazis, D., Leahy, R.M., 2011. Brainstorm: A User-Friendly Application for MEG/EEG Analysis. Comput. Intell. Neurosci. 2011, 1-13.

Tao, J.X., Baldwin, M., Hawes-Ebersole, S., Ebersole, J.S., 2007. Cortical Substrates of Scalp EEG Epileptiform Discharges. J. Clin. Neurophysiol. 24 (2), 96-100.

Traub, R.D., 1979. Neocortical pyramidal cells: a model with dendritic calcium conductance reproduces repetitive firing and epileptic behavior. Brain Res. 173 (2), 243-257.

Trujillo-Barreto, N.J., Aubert-Vázquez, E., Valdés-Sosa, P.A., 2004. Bayesian model averaging in EEG/MEG imaging. Neurolmage 21 (4), 1300-1319.

Tutorials/TutBEst - Brainstorm [WWW Document], 2015. . URL http://neuroimage.usc.edu/brainstorm/Tutorials/TutBEst

Uutela, K., Hämäläinen, M., Somersalo, E., 1999. Visualization of Magnetoencephalographic Data Using Minimum Current Estimates. Neurolmage 10 (2), 173-180.

von Ellenrieder, N., Beltrachini, L., Muravchik, C.H., Gotman, J., 2014. Extent of cortical generators visible on the scalp: Effect of a subdural grid. Neurolmage.

Wang, G., Ren, D., 2013. Effect of Brain-to-Skull Conductivity Ratio on EEG Source Localization Accuracy. BioMed Res. Int. 2013, e459346.

Wang, G., Worrell, G., Yang, L., Wilke, C., He, B., 2011. Interictal spike analysis of high-density EEG in patients with partial epilepsy. Clin. Neurophysiol. 122 (6), 1098-1105.

Wendling, F., 2005. Neurocomputational models in the study of epileptic phenomena. J. Clin. Neurophysiol. 22 (5), 285-287.

Wendling, F., Bellanger, J.J., Bartolomei, F., Chauvel, P., 2000. Relevance of nonlinear lumpedparameter models in the analysis of depth-EEG epileptic signals. Biol. Cybern. 83 (4), 367-378.

Wilson, H.R., Cowan, J.D., 1972. Excitatory and Inhibitory Interactions in Localized Populations of Model Neurons. Biophys. J. 12 (1), 1-24.

Wipf, D.P., Owen, J.P., Attias, H.T., Sekihara, K., Nagarajan, S.S., 2010. Robust Bayesian estimation of the location, orientation, and time course of multiple correlated neural sources using MEG. Neurolmage 49 (1), 641-655.

Yamazaki, M., Tucker, D.M., Fujimoto, A., Yamazoe, T., Okanishi, T., Yokota, T., Enoki, H., Yamamoto, T., 2012. Comparison of dense array EEG with simultaneous intracranial EEG for interictal spike detection and localization. Epilepsy Res. 98 (2-3), 166-173.

Yamazaki, M., Tucker, D.M., Terrill, M., Fujimoto, A., Yamamoto, T., 2013. Dense Array EEG Source Estimation in Neocortical Epilepsy. Front. Neurol. 4.

Zhu, M., Zhang, W., Dickens, D.L., Ding, L., 2014. Reconstructing spatially extended brain sources via enforcing multiple transform sparseness. Neurolmage 86, 280-293. 
Zhu, M., Zhang, W., Dickens, D.L., King, J.A., Ding, L., 2013. Sparse MEG Source Imaging For Reconstructing Dynamic Sources of Interictal Spikes in Partial Epilepsy: J. Clin. Neurophysiol. 30 (4), 313-328.

Figure 1: Spatio-temporal simulation protocol. (A) Source model (Geometry): Distributed source model providing spatial features of the spatio-temporal simulations where the source space was modeled by a dipolar layer distributed along the cortical surface and each dipole corresponds to a distinct neuronal population. (B) Source model (Activity): Simulation of temporal features using biophysical computational neural mass model where each neuronal population is made of two subsets of neurons: pyramidal cells (P) and local interneurons (I1, 12, 13). Pyramidal cells receive excitatory input (green arrows) from pyramidal cells of other populations and inhibitory input (red arrows) from interneurons. The interneurons only get excitatory input from the pyramidal cells. A connection from a given population $i$ to a population $I$ is characterized by a parameter $K_{i l}$ which represents the degree of coupling, which models the average density of action potentials fired by the pyramidal cells of one population as an excitatory input to the pyramidal cells of another population. Normal background and epileptic spike-like activities were obtained from two different settings of model parameters: excitatory and inhibitory gains in feedback loops, degree and direction of coupling between interconnected populations. These settings are used to simulate the time-course of "focal epileptic sources" (i.e. patches generating epileptic spikes) with surrounding normal background activity. Null coupling but increasing excitation/inhibition ratio generates epileptic spike activities. (C) Simulation pipeline: Assigning epileptic activity to the dipoles of the manually drawn extended source and background activity to all the dipoles on the mesh after grouping them into 1000 clusters. Then, application of the BEM forward model, to obtain simulated EEG and MEG signal.

Figure 2: Single Source analysis. EEG and MEG source localization results (with their corresponding validation metrics: AUC, SD and SE values) using CMEM and 4-ExSo-MUSIC on simulated sources of spatial extent $10 \mathrm{~cm}^{2}$ in (A) Basal temporal gyrus (BT), (B) Supplementary motor area (SMA), (C) Orbitofrontal (OF), (D) Central sulcus region (CS), and (E) Parietal source (P). All the source localization results were displayed over the inflated cortical surface obtained from Brainstorm software toolbox. In this figure, we presented source localization results of CMEM after an Otsu threshold, which is obtained by taking the absolute value of the current density at the peak of the spike, normalized to its maximum activity and thresholded upon the level of background activity (Otsu, 1979). We presented two maps for 4-ExSoMUSIC results: 4-ExSo-MUSIC metric map and the GOF thresholded binary map to be able to compare between the original and thresholded source maps. 
Figure 3: Plot of $A \cup C$ as a function of Signal-to-Noise Ratio (SNR) for all 200 single source localization results pooled together. (A) EEG source localization. (B) MEG source localization. Color code: CMEM in red color and 4-ExSo-MUSIC in blue. Black horizontal line showing $A U C=0.8$, considered a good level of detection accuracy.

Figure 4: Boxplot representation of AUC distribution for three simulated sources (BT, $P$ and $C S$ ) at 10 different source spatial extents $\left(0.5 \mathrm{~cm}^{2}, 1 \mathrm{~cm}^{2}, 2 \mathrm{~cm}^{2}, 3 \mathrm{~cm}^{2}, 4 \mathrm{~cm}^{2}, 5 \mathrm{~cm}^{2}, 7.5 \mathrm{~cm}^{2}, 10 \mathrm{~cm}^{2}, 20 \mathrm{~cm}^{2}\right.$, and $30 \mathrm{~cm}^{2}$ ) obtained over 20 simulated trials of the same configuration. (A) EEG source localization. (B) MEG source localization. The middle column represents the simulated source at $10 \mathrm{~cm}^{2}$ to display the regions on the cortical surface. Color code: CMEM in red color and 4-ExSo-MUSIC in blue, Cancellation index (IC) in grey ranging between 0 and 1, SNR in black ranging between 0 and 10 .

Figure 5: Boxplot representation of SD distribution for one simulated source (CS) at 10 different source spatial extents $\left(0.5 \mathrm{~cm}^{2}, 1 \mathrm{~cm}^{2}, 2 \mathrm{~cm}^{2}, 3 \mathrm{~cm}^{2}, 4 \mathrm{~cm}^{2}, 5 \mathrm{~cm}^{2}, 7.5 \mathrm{~cm}^{2}, 10 \mathrm{~cm}^{2}, 20 \mathrm{~cm}^{2}\right.$, and $\left.30 \mathrm{~cm}^{2}\right)$ obtained over 20 simulated trials of the same configuration. (A) EEG source localization. (B) MEG source localization. The middle column represents the simulated source at $10 \mathrm{~cm}^{2}$ to display the region on the cortical surface. Color code: CMEM in red color and 4-ExSo-MUSIC in blue.

Figure 6: Two source analysis: (A) Synchronous activities - Simulated sources of spatial extent $10 \mathrm{~cm}^{2}$ in temporal (patch 1) and parietal (patch 2) region. EEG source localization results (with their corresponding AUC values) using CMEM and 4-ExSo-MUSIC. MEG source localization results (with their corresponding AUC values) using CMEM and 4-ExSo-MUSIC. (B) Propagating activity - Simulated sources of spatial extent $10 \mathrm{~cm}^{2}$ in temporal region at peak 1 and parietal region at peak 2 after $20 \mathrm{~ms}$ of delay. EEG source localization results (with their corresponding AUC and SE values) using CMEM and 4-ExSo-MUSIC. MEG source localization results (with their corresponding AUC and SE values) using CMEM and 4-ExSoMUSIC. Here, we presented source localization results of CMEM after an Otsu threshold and for 4-ExSoMUSIC results the GOF thresholded binary map were presented.

Figure 7: Reconstructed temporal dynamics illustrated for a single trial of the propagating sources presented in Figure 5B. (A) Theoretical source dynamics showing the time course of every single dipole of the two epileptic patches generated by the neural mass model in a 10 s window consisting of four events (row 1 left) and a 450ms window segment extracted from the 10s window (row 1 right). (B) Simulated EEG signal extracted after marking the peaks of the two sources in the propagation pattern (row 2 left), 4-ExSo-MUSIC window for localizing temporal source (peak 1) is in pink box and window for localizing the propagated parietal source (peak 2) is in blue box; (row 2 right) shows the source dynamics reconstructed using 4-ExSo-MUSIC for the temporal and parietal sources in the respective windows; (row 3 left) shows the full 10s window of simulated EEG signal used for CMEM localization; (row 3 middle) shows the source dynamics reconstructed using CMEM for the full 10s window; (row 3 right) shows the zoomed time course reconstructed using cMEM. (C) Simulated MEG signal of the propagation pattern 
(row 4 left), 4-ExSo-MUSIC window for localizing temporal source (peak 1) is in pink box and window for localizing the propagated parietal source (peak 2) is in blue box; (row 4 right) shows the source dynamics reconstructed using 4-EXSo-MUSIC for the temporal and parietal sources; (row 5 left) shows the full 10s window of simulated MEG signal used for CMEM localization; (row 5 middle) shows the source dynamics reconstructed using CMEM for the full 10s window; (row 5 right) shows the zoomed time course reconstructed using cMEM.

Figure 8: Source localization results on MEG data of 26 averaged spikes recorded from patient 1 , displayed over the inflated cortical surface. (A) MEG signal and topography at the peak of the signal. (B) CMEM source localization results thresholded at $30 \%$ of the maximum amplitude, (C) 4-ExSo-MUSIC results thresholded using GOF criterion. (D) SLORETA results thresholded at $30 \%$ of the maximum amplitude. Pink color outline over the cortical surface represents the right orbitofrontal region showing maximal ictal and interictal activity in $\mathrm{iEEG}$ recordings.

Figure 9: Source localization results on EEG data of 85 averaged spikes recorded from patient 2, displayed over the inflated cortical surface. (A) MEG signal and topography at the peak of the signal. (B) cMEM source localization results thresholded at $30 \%$ of the maximum amplitude, (C) 4-ExSo-MUSIC results thresholded using GOF criterion. (D) SLORETA results thresholded at $30 \%$ of the maximum amplitude. Pink color outline over the cortical surface represents the lesion visible on MRI in this patient.

Supplementary Figure: Single Source analysis. EEG and MEG source localization results (with their corresponding validation metrics: AUC, SD and SE values) using cMEM and 4-ExSo-MUSIC on simulated sources of spatial extent $10 \mathrm{~cm}^{2}$ in (F) Insula, (G) Lateral orbito-frontal, (H) Temporal pole, (I) Superior Temporal region, and $(\mathrm{J})$ Occipital region. All the source localization results were displayed over the inflated cortical surface obtained from Brainstorm software toolbox. In this figure, we presented source localization results of CMEM after an Otsu threshold, which is obtained by taking the absolute value of the current density at the peak of the spike, normalized to its maximum activity and thresholded upon the level of background activity (Otsu, 1979). We presented two maps for 4-ExSo-MUSIC results: 4-ExSoMUSIC metric map and the GOF thresholded binary map to be able to compare between the original and thresholded source maps.

\section{Highlights}

- Comparison of cMEM and 4-ExSo-MUSIC on complex patterns of epileptic activity.

- High density EEG-MEG simulations using biophysical computational neural mass model.

- Both methods provide reliable detection accuracy of the source spatial extent.

- 4-ExSo-MUSIC better for single and deep sources with large signal-to-noise ratio.

- cMEM provides better results for complex spatio-temporal patterns 


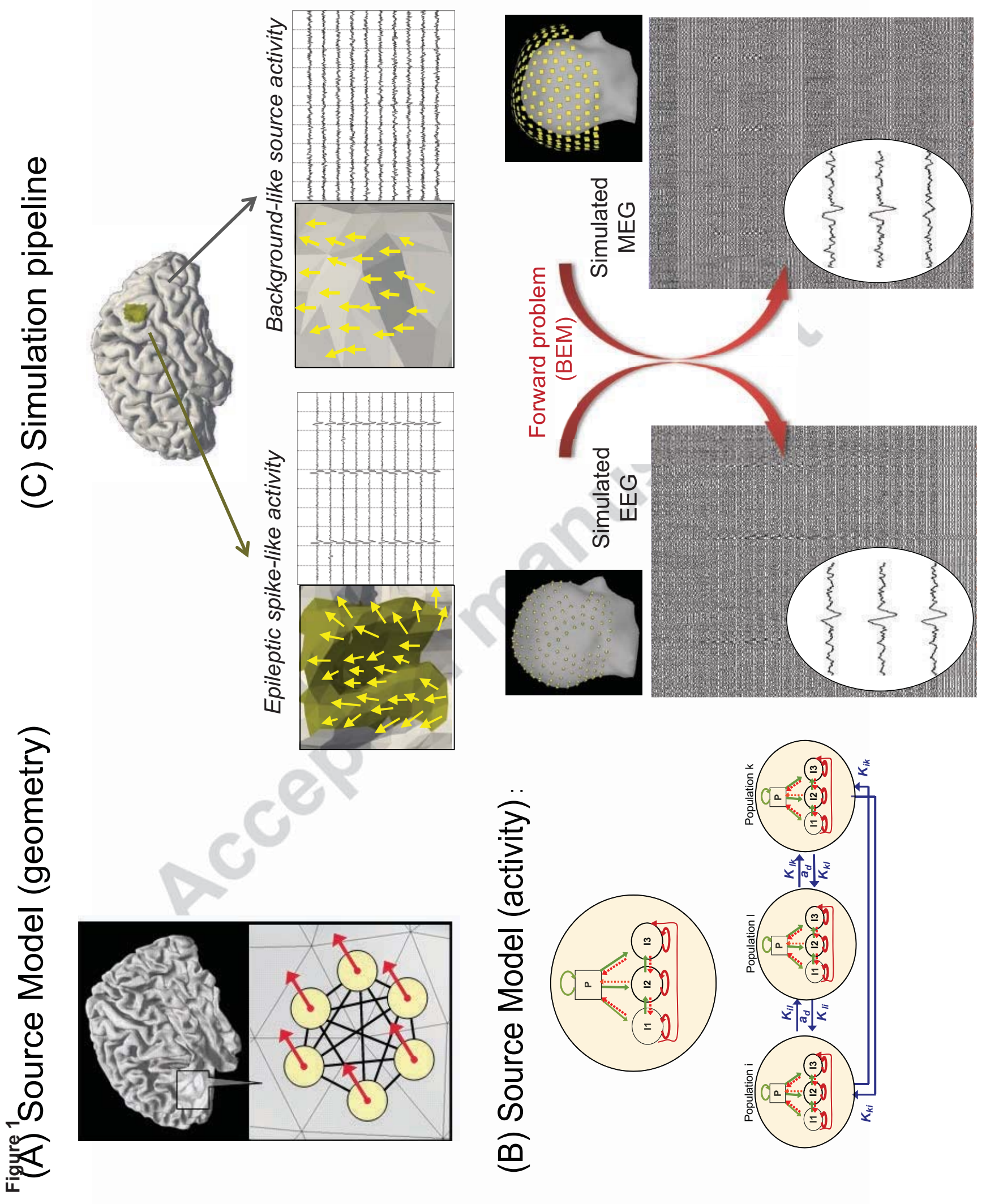



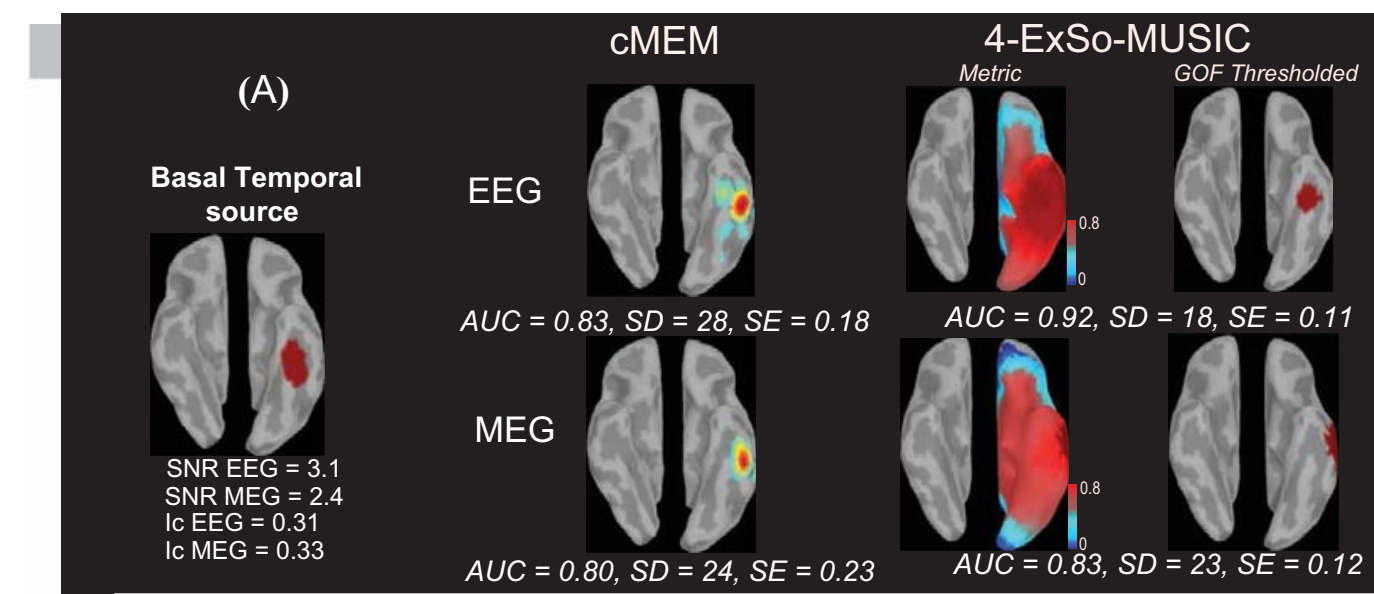

$A \cup C=0.83, S D=28, S E=0.18$

MEG
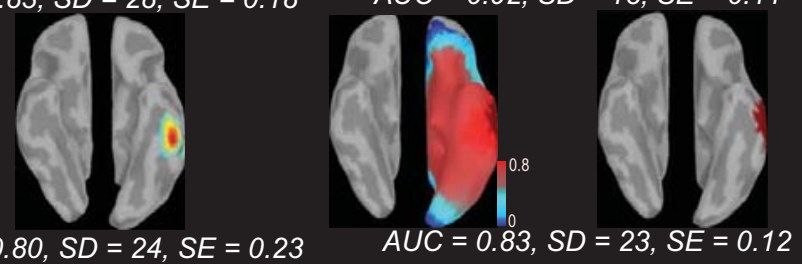

(B)

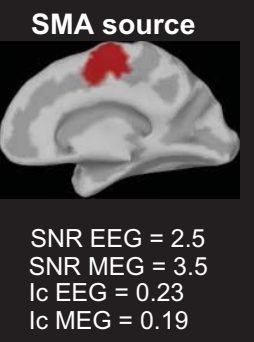

$A U C=0.80, S D=24, S E=0.23$

$A U C=0.83, S D=23, S E=0.12$
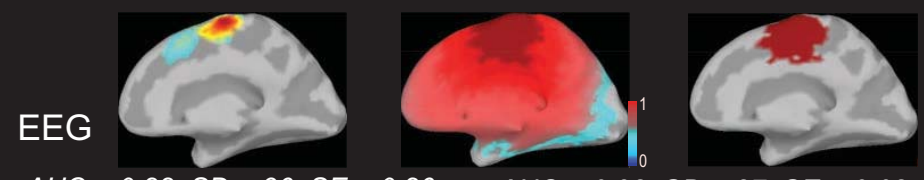

$A \cup C=0.88, S D=36, S E=0.26$

$A U C=0.98, S D=27, S E=0.08$

MEG
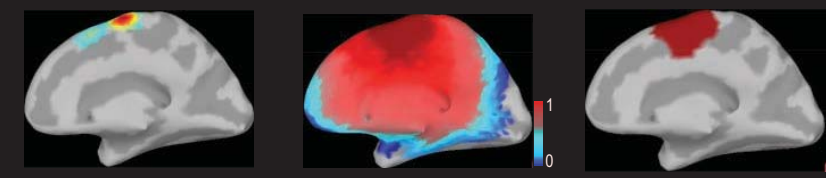

$A U C=0.84, S D=35, S E=0.18$

$A U C=0.91, S D=31, S E=0.05$

(C)

Orbitofrontal source

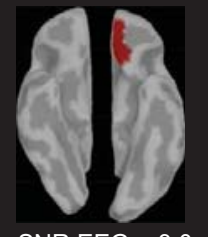

SNR EEG $=3.0$

SNR MEG $=1.8$

Ic EEG $=0.20$

Ic $M E G=0.23$
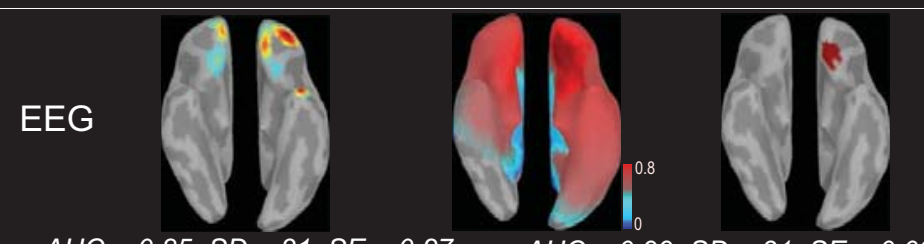

$A U C=0.85, S D=31, S E=0.27$

$A \cup C=0.99, S D=31, S E=0.09$

MEG
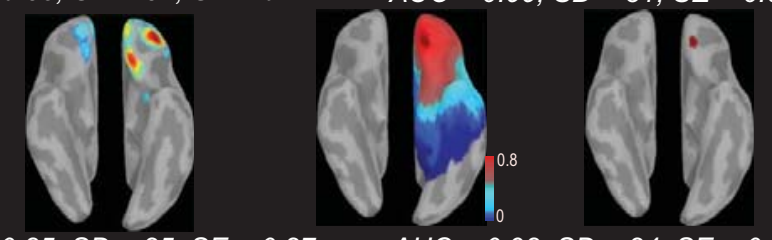

$A \cup C=0.85, S D=25, S E=0.27$

$A \cup C=0.98, S D=24, S E=0.10$

(D)

Central Sulcus source

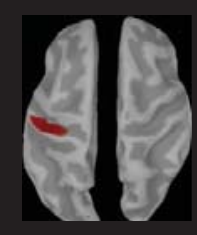

SNR EEG $=4.7$

SNR MEG $=4.9$

Ic EEG $=0.23$

Ic MEG $=0.20$
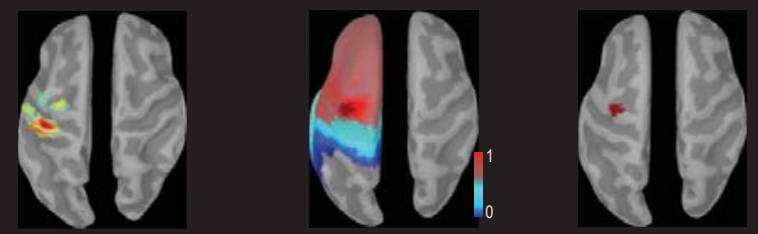

$A \cup C=0.90, S D=10, S E=0.19$

$A U C=0.71, S D=18, S E=0.06$

MEG
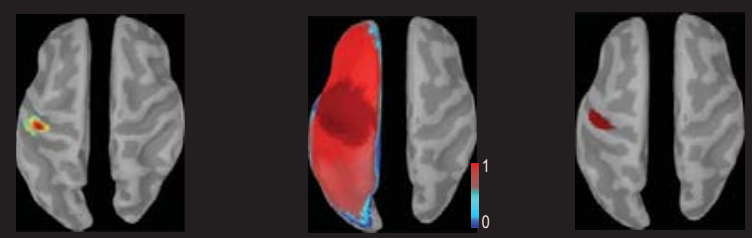

$A U C=0.86, S D=7, S E=0.18$

$A \cup C=0.86, S D=15, S E=0.05$

(E)

Parietal source

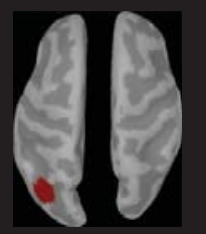

EEG
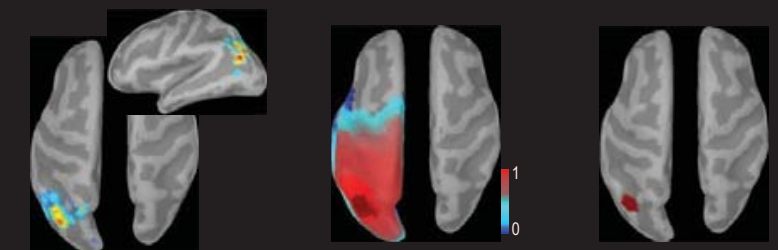

$A U C=0.86, S D=10, S E=0.16$

$A \cup C=0.97, S D=27, S E=0.08$

MEG

SNR EEG $=3.1$

SNR MEG $=1.6$

Ic $\mathrm{EEG}=0.40$

Ic MEG $=0.50$
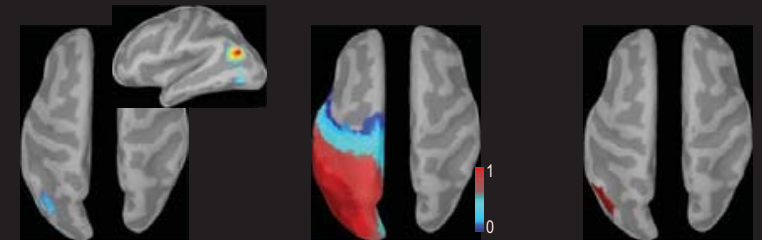


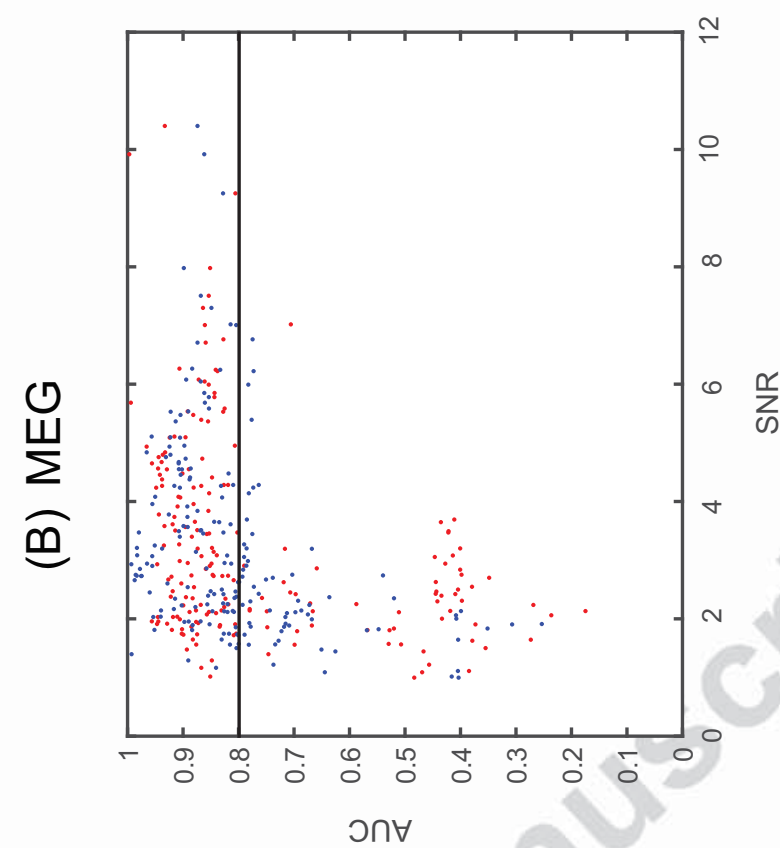

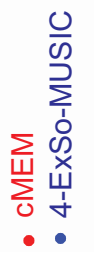

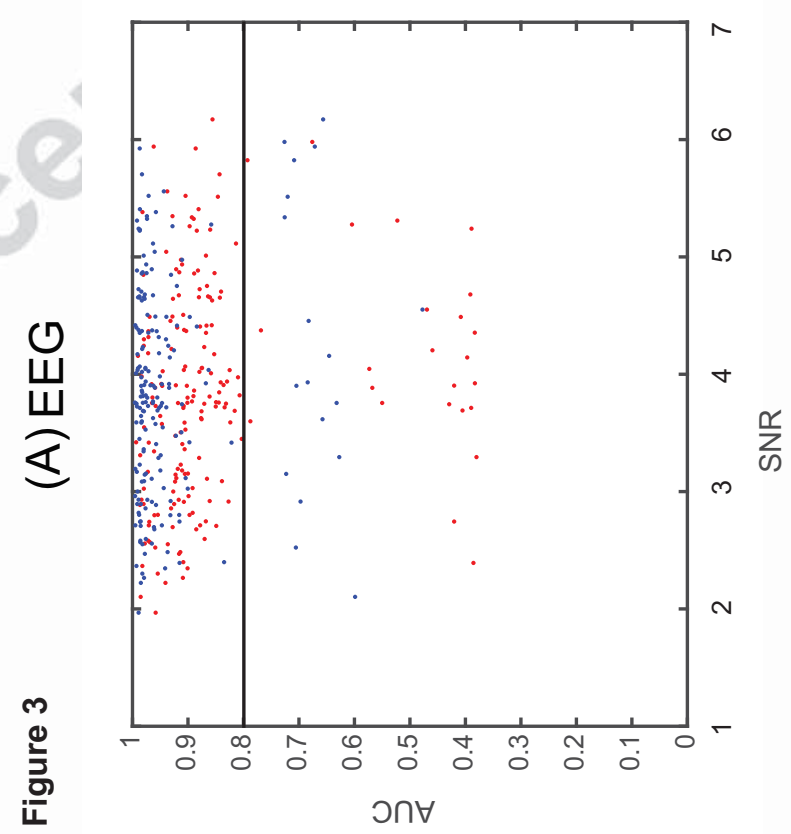



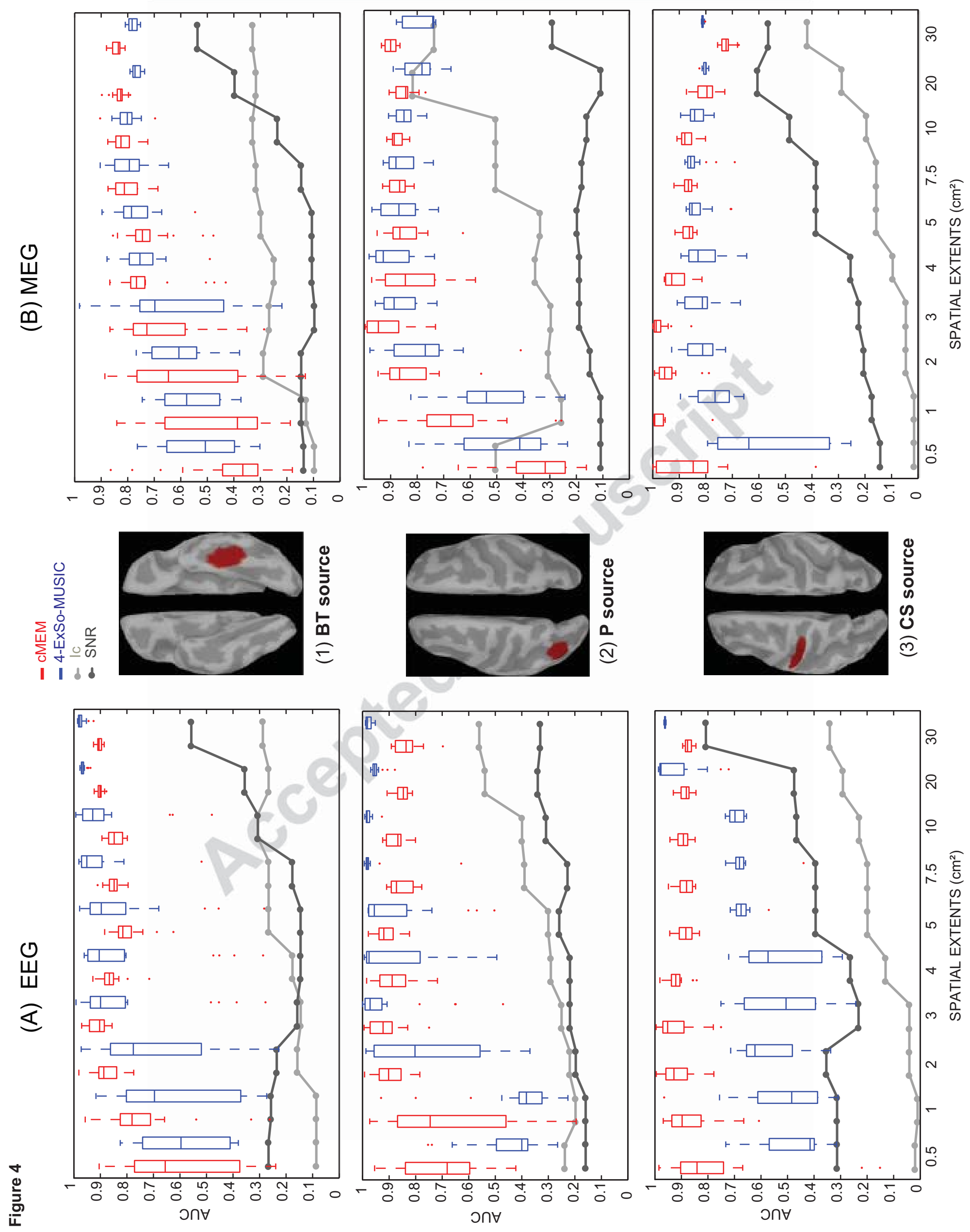


\section{ACCEPTED MANUSCRIPT}
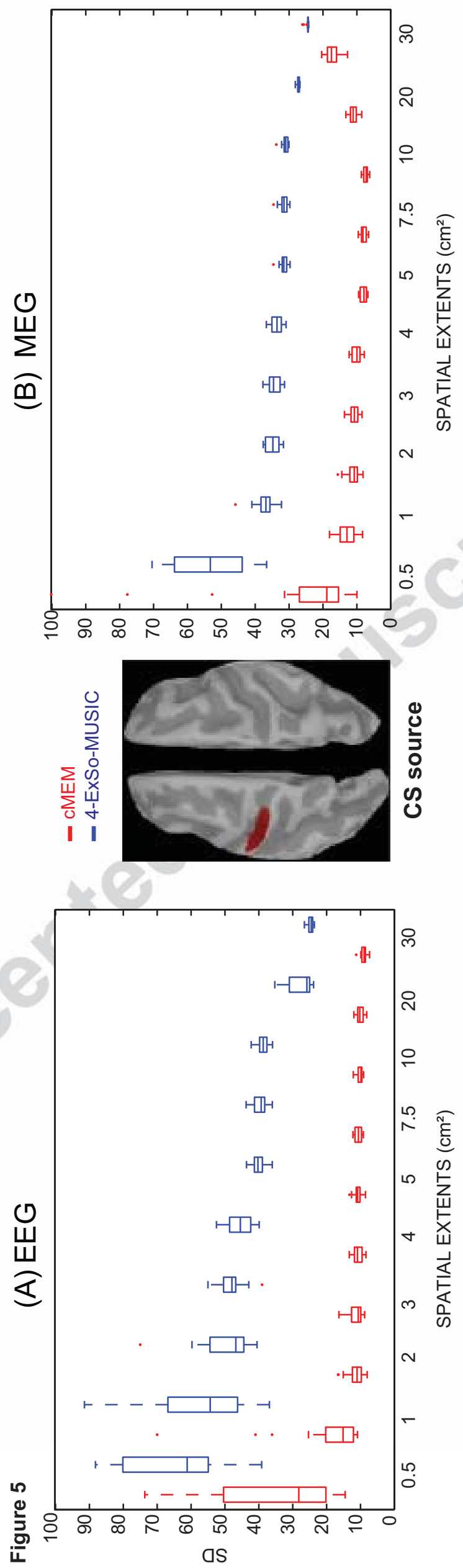

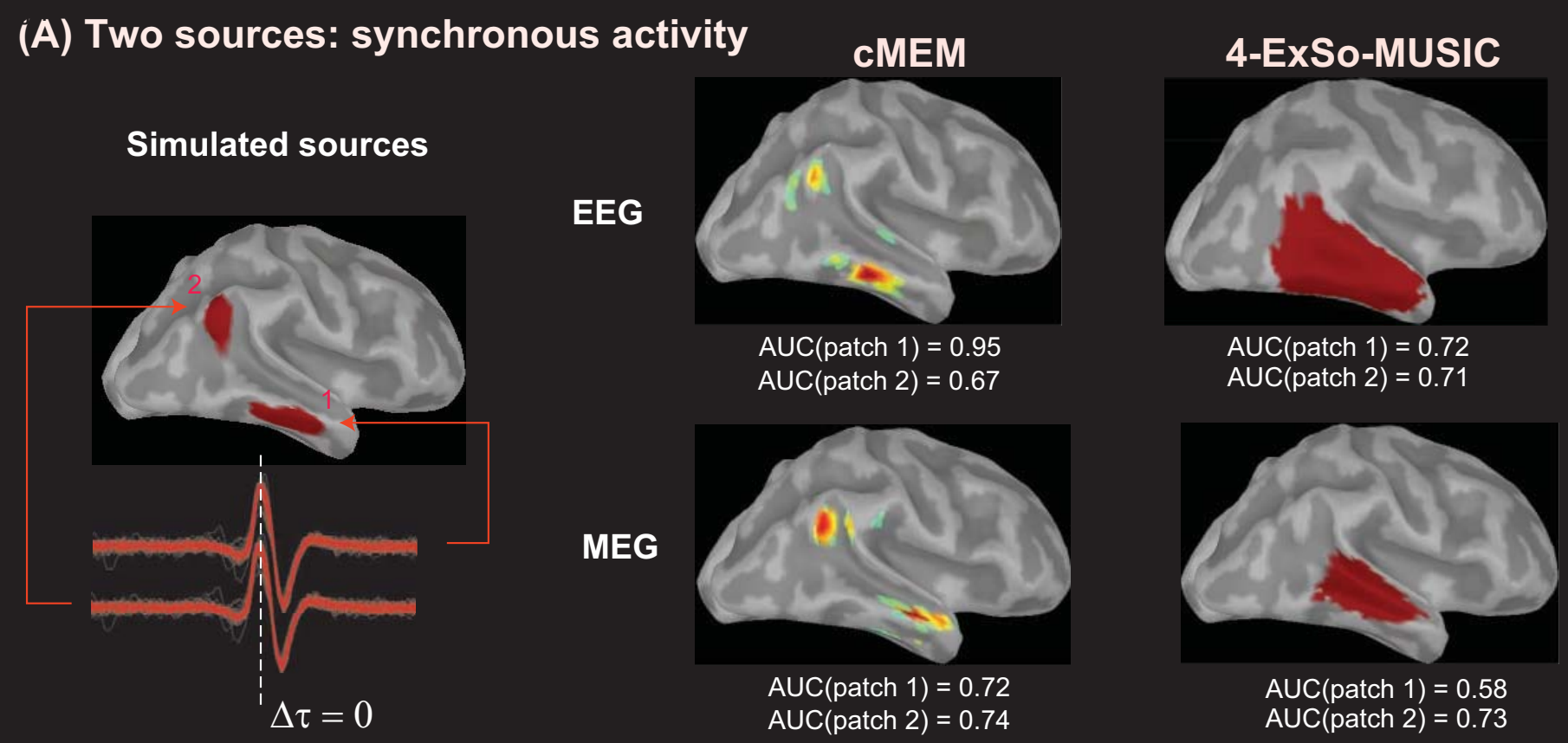

(B) Two sources : propagating activity

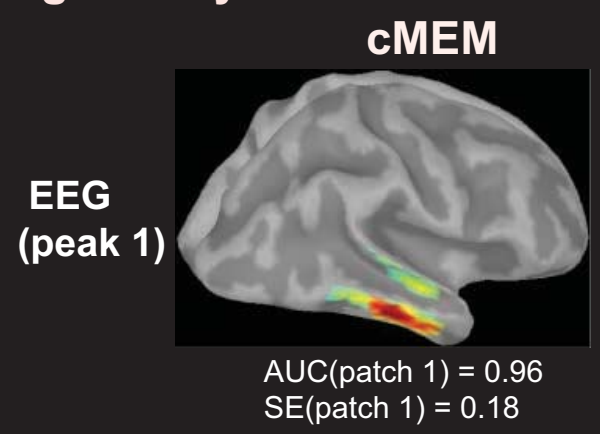

\section{Simulated sources}
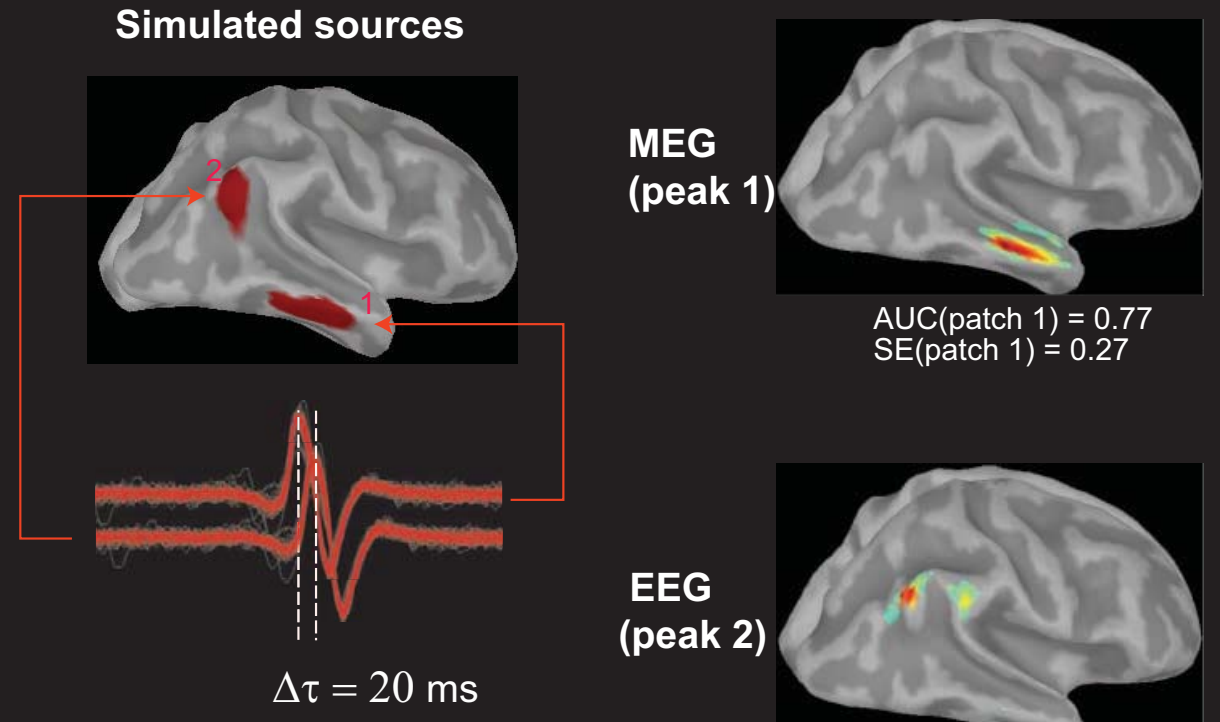

$\mathrm{AUC}($ patch 1) $=0.77$

$\mathrm{SE}($ patch 1$)=0.27$
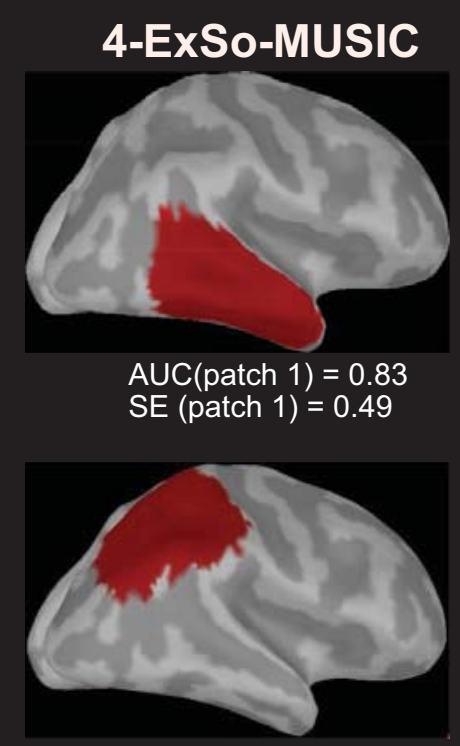

AUC $($ patch 1) $=0.47$

SE $($ patch 1) $=0.68$
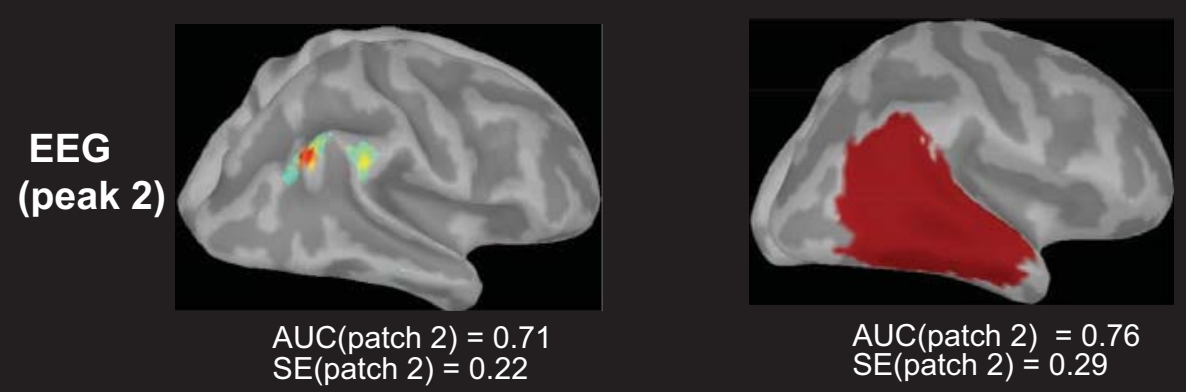

AUC (patch 2) $=0.76$ $\mathrm{SE}($ patch 2) $=0.29$
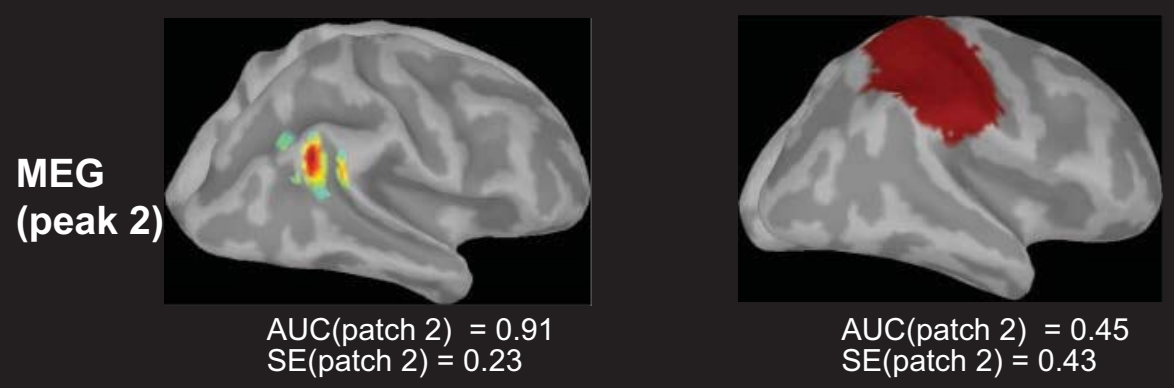
(A) Simulated source dynamics

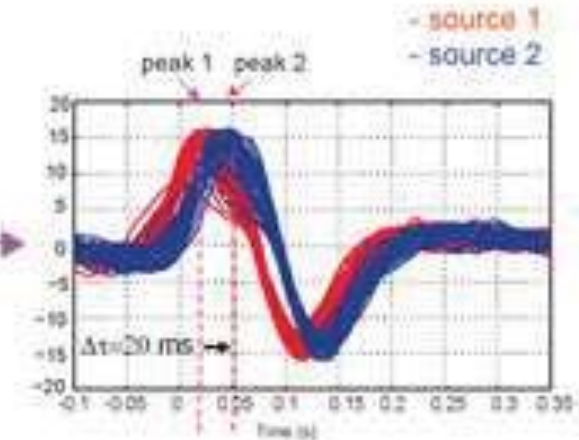

(B) EEG source reconstruction
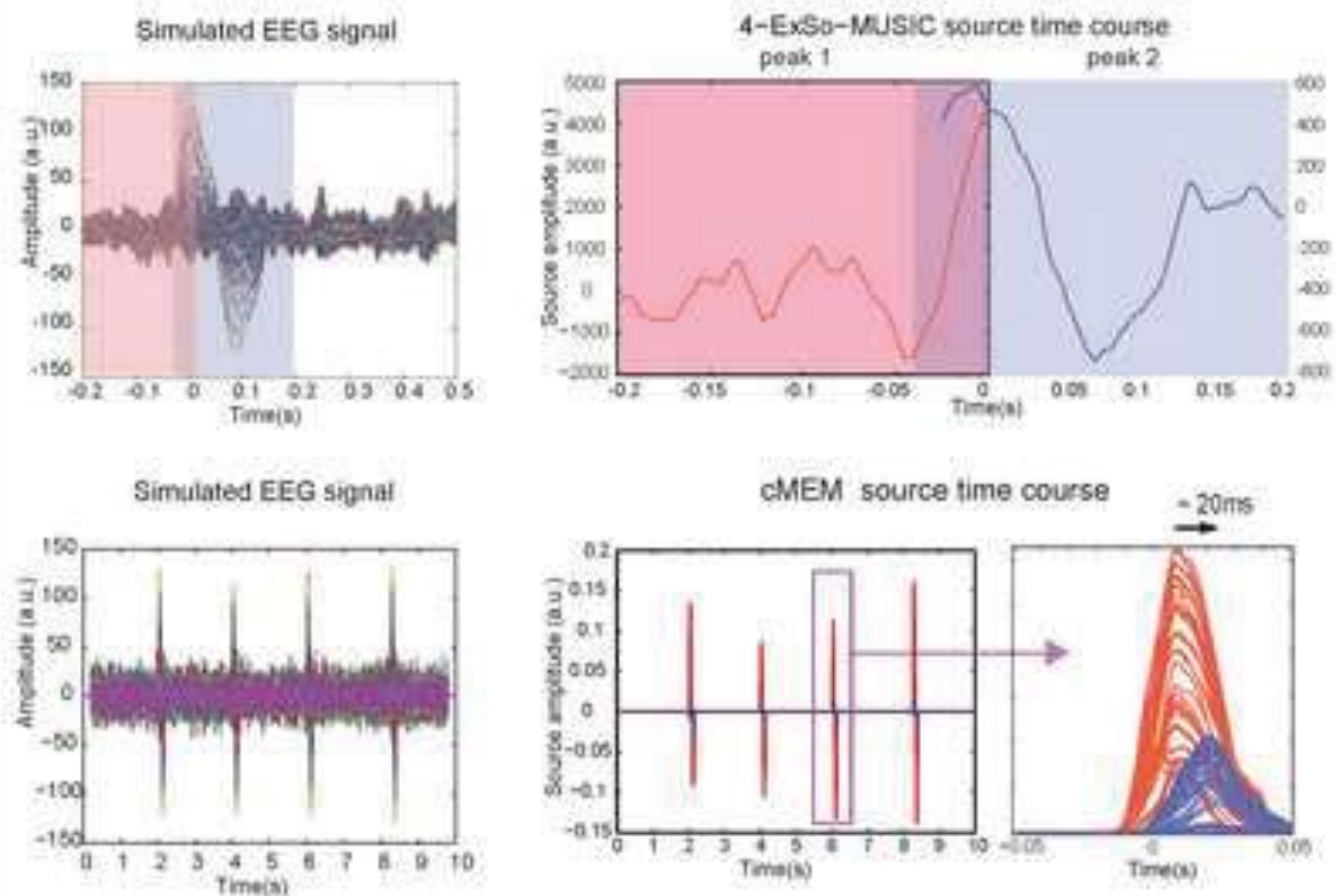

\section{(C) MEG source reconstruction}
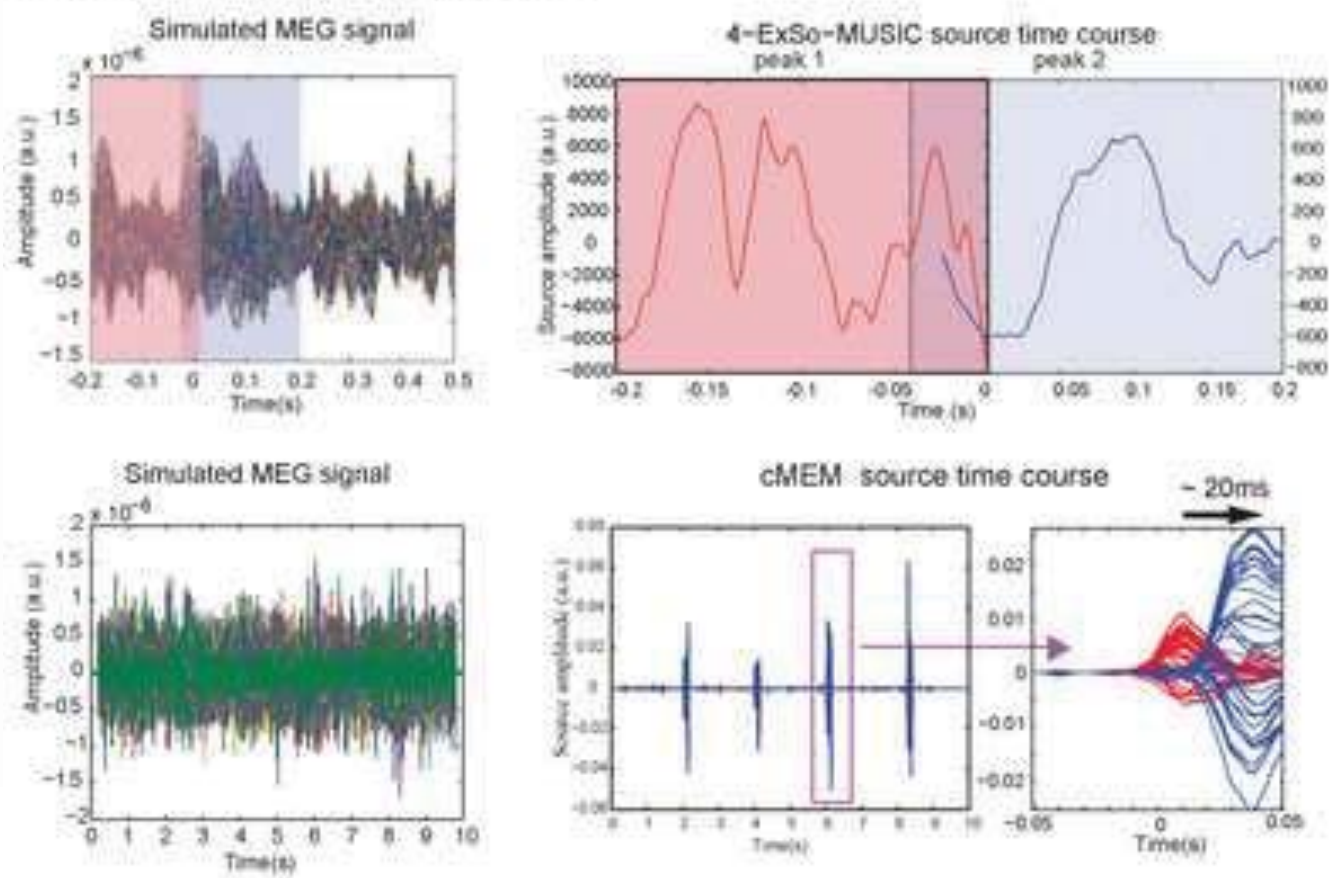

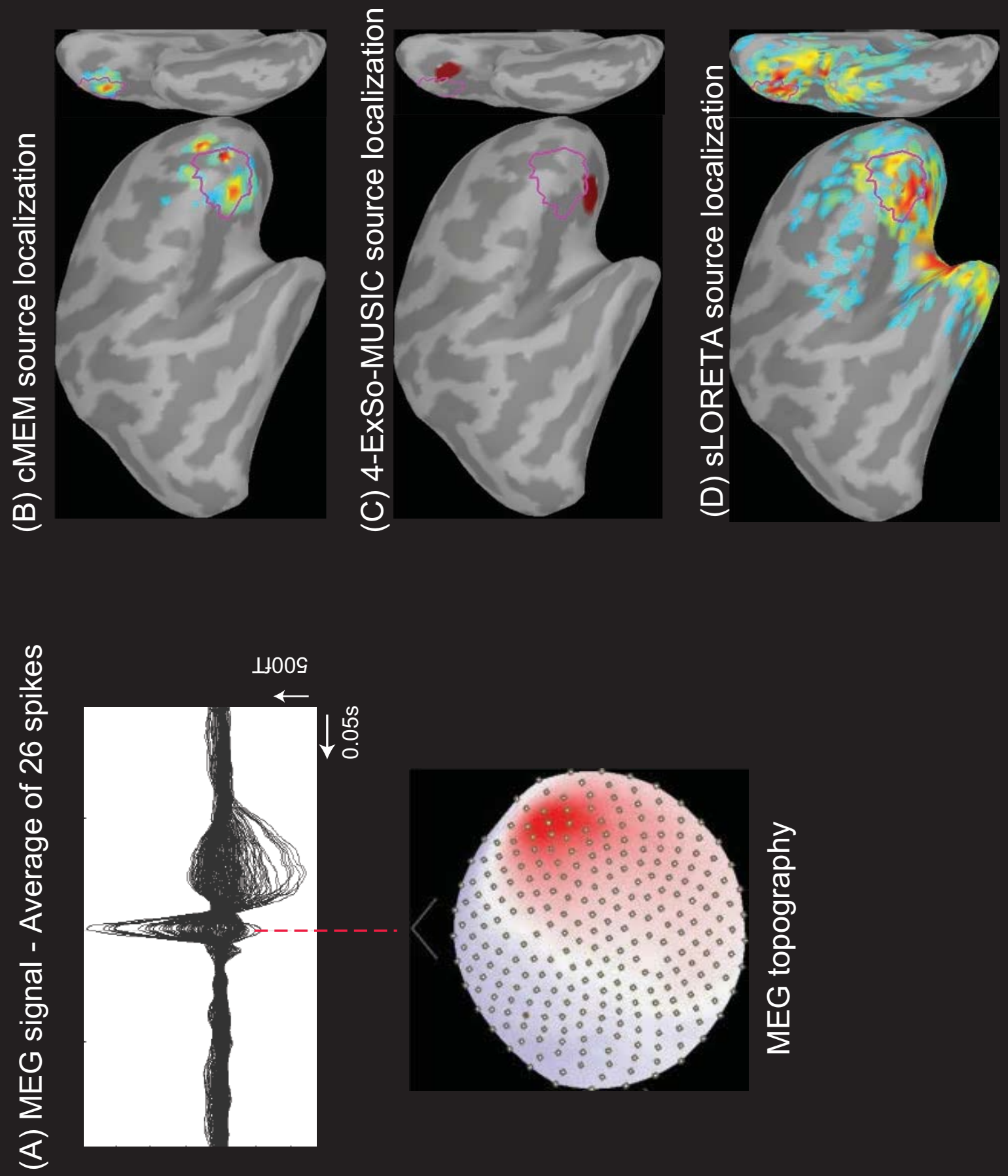


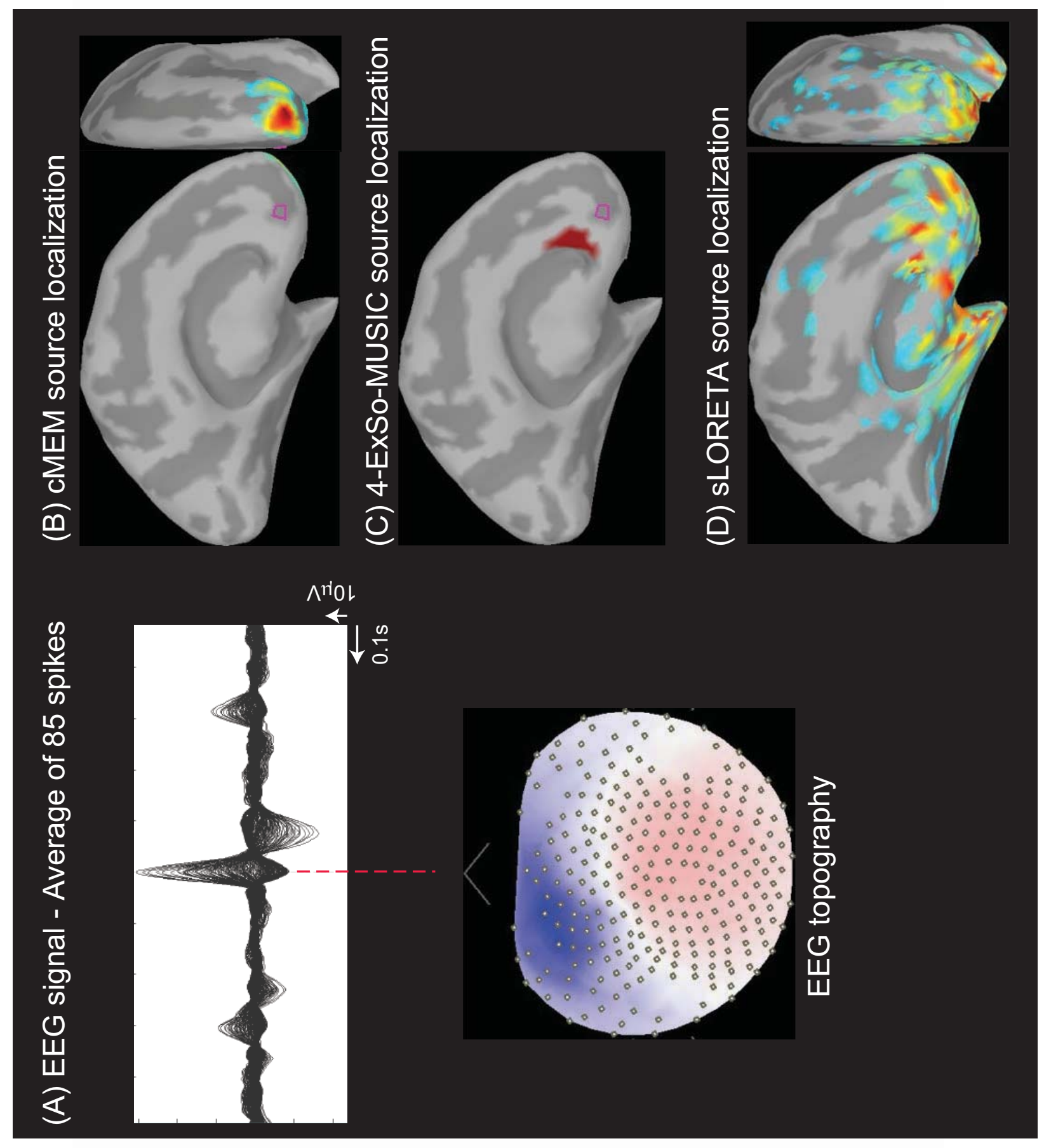

